\title{
Midpoint Inequalities in Fractional Calculus Defined Using Positive Weighted Symmetry Function Kernels
}

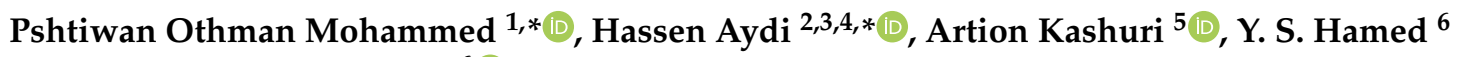 \\ and Khadijah M. Abualnaja ${ }^{6}$ (D) \\ 1 Department of Mathematics, College of Education, University of Sulaimani, Sulaimani 46001, \\ Kurdistan Region, Iraq \\ 2 Institut Supérieur d'Informatique et des Techniques de Communication, Université de Sousse, \\ Hammam Sousse 4000, Tunisia \\ 3 Department of Mathematics and Applied Mathematics, Sefako Makgatho Health Sciences University, \\ Ga-Rankuwa, South Africa \\ 4 China Medical University Hospital, China Medical University, Taichung 40402, Taiwan \\ 5 Department of Mathematics, Faculty of Technical Science, University Ismail Qemali, Vlora 9401, Albania; \\ artionkashuri@gmail.com \\ 6 Department of Mathematics and Statistics, College of Science, Taif University, P.O. Box 11099, \\ Taif 21944, Saudi Arabia; yasersalah@tu.edu.sa (Y.S.H.); Kh.abualnaja@tu.edu.sa (K.M.A.) \\ * Correspondence: pshtiwansangawi@gmail.com (P.O.M.); hassen.aydi@isima.rnu.tn (H.A.)
}

Citation: Mohammed, P.O.; Aydi, H.; Kashuri, A.; Hamed, Y.S.;

Abualnaja, K.M. Midpoint Inequalities in Fractional Calculus Defined Using Positive Weighted Symmetry Function Kernels. Symmetry 2021, 13, 550. https:// doi.org/10.3390/sym13040550

Academic Editor: Aviv Gibali

Received: 25 February 2021

Accepted: 24 March 2021

Published: 26 March 2021

Publisher's Note: MDPI stays neutral with regard to jurisdictional claims in published maps and institutional affiliations.

\section{Copyright: (c) 2021 by the authors.} Licensee MDPI, Basel, Switzerland. This article is an open access article distributed under the terms and conditions of the Creative Commons Attribution (CC BY) license (https:/ / creativecommons.org/licenses/by/ $4.0 /)$.

\begin{abstract}
The aim of our study is to establish, for convex functions on an interval, a midpoint version of the fractional HHF type inequality. The corresponding fractional integral has a symmetric weight function composed with an increasing function as integral kernel. We also consider a midpoint identity and establish some related inequalities based on this identity. Some special cases can be considered from our main results. These results confirm the generality of our attempt.
\end{abstract}

Keywords: symmetry; weighted fractional operators; convex functions; HHF type inequality

\section{Introduction}

Let $\mathcal{J} \subset \mathcal{R}$ be an interval and let $\mathrm{u}: \mathcal{J} \rightarrow \mathcal{R}$ be a continuous function. Then, the function $u$ is called convex if it satisfies

$$
\mathrm{u}\left(\kappa \mathbf{c}_{1}+(1-\kappa) \mathbf{c}_{2}\right) \leq \kappa \mathrm{u}\left(\mathbf{c}_{1}\right)+(1-\kappa) \mathrm{u}\left(\mathbf{c}_{2}\right), \quad \forall \mathbf{c}_{1}, \mathbf{c}_{2} \in \mathcal{J} \text { and } \kappa \in[0,1]
$$

The function $u$ is called concave whenever $-\mathrm{u}$ is convex.

For convex functions $\mathrm{u}: \mathcal{J} \rightarrow \mathcal{R}$, there is an important integral inequality in the literature, namely the Hermite-Hadamard or, briefly, the HH integral inequality, which is given by [1]:

$$
\mathrm{u}\left(\frac{\mathrm{c}_{1}+\mathrm{c}_{2}}{2}\right) \leq \frac{1}{\mathrm{c}_{2}-\mathrm{c}_{1}} \int_{\mathrm{c}_{1}}^{\mathrm{c}_{2}} \mathrm{u}(x) d x \leq \frac{\mathrm{u}\left(\mathrm{c}_{1}\right)+\mathrm{u}\left(\mathrm{c}_{2}\right)}{2}
$$

where $c_{1}<c_{2}$ belong to $\mathcal{J}$. In the literature, one can observe that the HH integral inequality (2) has been applied to different classes of convexity such as $G A$-convexity [2], quasi-convexity [3,4], s-convexity [5], $(\alpha, m)$-convexity [6], exponentially convexity [7,8], $M T$-convexity [9], and the readers can consult [10,11] to find other types.

As we know, fractional calculus is a generalized form of integer order calculus. Various forms of fractional derivatives including RL, Hadamard, Caputo, Caputo-Hadamard, Riesz, $\psi-R L$, Prabhakar, and weighted versions [12-16] have been developed to date. Most of these versions are described in the RL sense based on the corresponding fractional integral. Many integer-order integral inequalities such as Ostrowski [17], Simpson [18], 
Hardy [19], Olsen [20], Gagliardo-Nirenberg [21], Opial [22,23] and Rozanova [24] have been generalized and reformulated from the fractional point of view.

In addition, in 2013, the HH integral inequality (2) was generalized and reformulated by Sarikaya et al. [25] in terms of RL fractional integrals. Their result is given by:

$$
\mathrm{u}\left(\frac{\mathbf{c}_{1}+\mathbf{c}_{2}}{2}\right) \leq \frac{\Gamma(v+1)}{2\left(\mathbf{c}_{2}-\mathbf{c}_{1}\right)^{v}}\left[{ }^{R L} \mathcal{I}_{\mathbf{c}_{1}+}^{v} \mathrm{u}\left(\mathbf{c}_{2}\right)+{ }^{R L} \mathcal{I}_{\mathbf{c}_{2}-}^{v} \mathrm{u}\left(\mathbf{c}_{1}\right)\right] \leq \frac{\mathrm{u}\left(\mathbf{c}_{1}\right)+\mathrm{u}\left(\mathbf{c}_{2}\right)}{2}
$$

where $\mathrm{u}: \mathcal{J} \rightarrow \mathcal{R}$ is assumed to be a positive convex function, continuous on the closed interval $\left[\mathbf{c}_{1}, \mathbf{c}_{2}\right]$, and for Lebesgue, almost all $x \in\left[\mathbf{c}_{1}, \mathbf{c}_{2}\right]$ when $\mathrm{u}(x) \in L^{1}\left[\mathbf{c}_{1}, \mathbf{c}_{2}\right]$ with $\mathbf{c}_{1}<\mathbf{c}_{2}$, where ${ }^{R L} \mathcal{I}_{\mathbf{c}_{1}+}^{v}$ and ${ }^{R L} \mathcal{I}_{\mathbf{c}_{2}-}^{v}$ are the left- and right-sided RL fractional integrals of order $v>0$, defined by [12]:

$$
\begin{aligned}
& { }^{R L} \mathcal{I}_{\mathbf{c}_{1}+}^{v} \mathrm{u}(x)=\frac{1}{\Gamma(v)} \int_{\mathbf{c}_{1}}^{x}(x-\kappa)^{\nu-1} \mathbf{u}(\kappa) d \kappa, \quad x>\mathbf{c}_{1} ； \\
& { }^{R L} \mathcal{I}_{\mathbf{c}_{2}-\mathbf{u}}^{v}(x)=\frac{1}{\Gamma(v)} \int_{x}^{\mathbf{c}_{2}}(\kappa-x)^{v-1} \mathbf{u}(\kappa) d \kappa, \quad x<\mathbf{c}_{2},
\end{aligned}
$$

respectively.

The inequality (3) is also known as the endpoint $\mathrm{HH}$ inequality due to using the ends $\mathbf{c}_{1}, \mathbf{c}_{2}$ of the interval.

On the other hand, the endpoint HH inequality (3) has been applied for various classes of convexity such as $\lambda_{\psi}$-convexity [26], F-convexity [27], $(\alpha, m)$-convexity [28], MT-convexity [29]. The reader can find other types of convexity in the literature, which in particular, is true for [30]. In the mean time, applying the end-point HH inequality to other models of fractional calculus has received a huge amount of attention. For example, this is true for RL fractional models [31], conformable fractional models [32,33], generalized fractional models [34], $\psi$ RL fractional models [35,36], tempered fractional models [37], and $A B$ - and Prabhakar fractional models [38].

After extending the important field of the integral inequalities in (2) and (3), a new version of the endpoint $\mathrm{HH}$ inequality (3) was found by Sarikaya and Yildirim [39], namely the midpoint $\mathrm{HH}$ inequality due to using the midpoint $\frac{\mathbf{c}_{1}+\mathbf{c}_{2}}{2}$ of the interval, which is given by

$$
\mathrm{u}\left(\frac{\mathbf{c}_{1}+\mathbf{c}_{2}}{2}\right) \leq \frac{2^{v-1} \Gamma(v+1)}{\left(\mathbf{c}_{2}-\mathbf{c}_{1}\right)^{v}}\left[{ }^{R L} \mathcal{I}_{\left(\frac{\mathbf{c}_{1}+\mathbf{c}_{2}}{2}\right)+}^{v} \mathrm{u}\left(\mathbf{c}_{2}\right)+{ }^{R L} \mathcal{I}_{\left(\frac{\mathbf{c}_{1}+\mathbf{c}_{2}}{2}\right)-}^{v} \mathrm{u}\left(\mathbf{c}_{1}\right)\right] \leq \frac{\mathrm{u}\left(\mathbf{c}_{1}\right)+\mathrm{u}\left(\mathbf{c}_{2}\right)}{2},
$$

where the function $\mathrm{u}:\left[\mathbf{c}_{1}, \mathbf{c}_{2}\right] \rightarrow \mathcal{R}$ is convex and continuous.

Definition 1 ([40]). Let $g:\left[c_{1}, c_{2}\right] \rightarrow[0, \infty)$ be a function. Then, we say $g$ is symmetric with respect to $\left(c_{1}+c_{2}\right) / 2$ if

$$
g\left(c_{1}+c_{2}-x\right)=g(x), \quad \forall x \in\left[c_{1}, c_{2}\right]
$$

Based on above definition, in [41], Fejér found a new extension of the HH type inequality (2), namely the HHF type inequality, and the result is as follows:

$$
\mathrm{u}\left(\frac{\mathbf{c}_{1}+\mathbf{c}_{2}}{2}\right) \int_{\mathbf{c}_{1}}^{\mathbf{c}_{2}} g(x) d x \leq \int_{\mathbf{c}_{1}}^{\mathbf{c}_{2}} \mathbf{u}(x) g(x) d x \leq \frac{\mathbf{u}\left(\mathbf{c}_{1}\right)+\mathbf{u}\left(\mathbf{c}_{2}\right)}{2} \int_{\mathbf{c}_{1}}^{\mathbf{c}_{2}} g(x) d x,
$$


where $g$ is the integrable function, and Işcan [42] found the endpoint version of (7) in the sense of RL fractional integrals, which is also the extension of (3). The result is as follows:

$$
\begin{aligned}
& \mathrm{u}\left(\frac{\mathbf{c}_{1}+\mathbf{c}_{2}}{2}\right)\left[{ }^{R L} \mathcal{I}_{\mathbf{c}_{1}+}^{v} g\left(\mathbf{c}_{2}\right)+{ }^{R L} \mathcal{I}_{\mathbf{c}_{2}-}^{v} g\left(\mathbf{c}_{1}\right)\right] \leq\left[{ }^{R L} \mathcal{I}_{\mathbf{c}_{1}+}^{v}(\mathrm{u} g)\left(\mathbf{c}_{2}\right)+{ }^{R L} \mathcal{I}_{\mathbf{c}_{2}-}^{v}(\mathrm{ug})\left(\mathbf{c}_{1}\right)\right] \\
& \leq \frac{\mathrm{u}\left(\mathbf{c}_{1}\right)+\mathrm{u}\left(\mathbf{c}_{2}\right)}{2}\left[{ }^{R L} \mathcal{I}_{\mathbf{c}_{1}+g}^{v}\left(\mathbf{c}_{2}\right)+{ }^{R L} \mathcal{I}_{\mathbf{c}_{2}-g}^{v}\left(\mathbf{c}_{1}\right)\right]
\end{aligned}
$$

where $u$ is convex and continuous and the function $g$ belongs to $L^{1}\left[\mathbf{c}_{1}, \mathbf{c}_{2}\right]$ and is symmetric (see Definition 1).

It is worth mentioning that the midpoint version of (8) has not been found yet, even though many related inequalities of midpoint type were obtained in [43].

Recently, Mohammed et al. [44] found a new endpoint HHF-inequality in terms of weighted fractional integrals with positive weighted symmetric function in a kernel, and their result is as follows:

$$
\begin{aligned}
& \mathrm{u}\left(\frac{\mathbf{c}_{1}+\mathbf{c}_{2}}{2}\right)\left[\left(\varrho^{-1}\left(\mathbf{c}_{1}\right)+\mathcal{I}^{v: \varrho}(w \circ \varrho)\right)\left(\varrho^{-1}\left(\mathbf{c}_{2}\right)\right)+\left(\mathcal{I}_{\varrho^{-1}\left(\mathbf{c}_{2}\right)-}^{v: \varrho}(w \circ \varrho)\right)\left(\varrho^{-1}\left(\mathbf{c}_{1}\right)\right)\right] \\
& \leq w\left(\mathbf{c}_{2}\right)\left({ }_{\varrho^{-1}\left(\mathbf{c}_{1}\right)+} \mathcal{I}_{w \circ \varrho}^{v: \varrho}(\mathbf{u} \circ \varrho)\right)\left(\varrho^{-1}\left(\mathbf{c}_{2}\right)\right)+w\left(\mathbf{c}_{1}\right)\left({ }_{w \circ \varrho^{\prime}} \mathcal{I}_{\varrho^{-1}\left(\mathbf{c}_{2}\right)-}^{v: \varrho}(\mathbf{u} \circ \varrho)\right)\left(\varrho^{-1}\left(\mathbf{c}_{1}\right)\right) \\
& \leq \frac{\mathrm{u}\left(\mathbf{c}_{1}\right)+\mathrm{u}\left(\mathbf{c}_{2}\right)}{2}\left[\left(\varrho^{-1}\left(\mathbf{c}_{1}\right)+\mathcal{I}^{v: \varrho}(w \circ \varrho)\right)\left(\varrho^{-1}\left(\mathbf{c}_{2}\right)\right)\right. \\
& \left.+\left(\mathcal{I}_{\varrho^{-1}\left(\mathbf{c}_{2}\right)-}^{v: \varrho}(w \circ \varrho)\right)\left(\varrho^{-1}\left(\mathbf{c}_{1}\right)\right)\right] .
\end{aligned}
$$

Here, $\mathrm{u}$ is a convex and continuous function, $\varrho(x)$ a monotone increasing function from the interval $\left(\mathbf{c}_{1}, \mathbf{c}_{2}\right]$ onto itself with a continuous derivative $\varrho^{\prime}(x)$ on the open interval $\left(\mathbf{c}_{1}, \mathbf{c}_{2}\right)$, and $w:\left[\mathbf{c}_{1}, \mathbf{c}_{2}\right] \rightarrow(0, \infty)$ is an integrable function, which is symmetric with respect to $\left(\mathbf{c}_{1}+\mathbf{c}_{2}\right) / 2$, where $\mathbf{c}_{1}<\mathbf{c}_{2}$.

Definition 2. Let $\left(c_{1}, c_{2}\right) \subseteq \mathcal{R}$ and $\varrho(x)$ be an increasing positive and monotone function on the interval $\left(c_{1}, c_{2}\right]$ with a continuous derivative $\varrho^{\prime}(x)$ on the open interval $\left(c_{1}, c_{2}\right)$. Then, the left-sided and right-sided the weighted fractional integrals of a function $\mathrm{u}$ according to another function $\varrho(x)$ on $\left[\boldsymbol{c}_{1}, \boldsymbol{c}_{2}\right]$ are defined by [15]:

$$
\begin{aligned}
& \left({ }_{c_{1}+} \mathcal{I}_{w}^{v: \varrho} \mathrm{u}\right)(x)=\frac{[w(x)]^{-1}}{\Gamma(v)} \int_{c_{1}}^{x} \varrho^{\prime}(\kappa)(\varrho(x)-\varrho(\kappa))^{v-1} \mathrm{u}(\kappa) w(\kappa) d \kappa, \\
& \left({ }_{w} \mathcal{I}_{c_{2}-}^{v: \varrho} \mathrm{u}\right)(x)=\frac{[w(x)]^{-1}}{\Gamma(v)} \int_{x}^{c_{2}} \varrho^{\prime}(\kappa)(\varrho(\kappa)-\varrho(x))^{v-1} \mathrm{u}(\kappa) w(\kappa) d \kappa, \quad v>0,
\end{aligned}
$$

for $[w(x)]^{-1}:=\frac{1}{w(x)}$ such that $w(x) \neq 0$.

Remark 1. From Definition 2, we can obtain the following special cases.

- If $\varrho(x)=x$ and $w(x)=1$, then the weighted fractional integrals (10) reduce to the classical RL fractional integrals (4).

- If $w(x)=1$, we obtain the fractional integrals of the function u with respect to the function $\varrho(x)$, which is defined by $[13,14]$ :

$$
\begin{aligned}
\left(c_{1}+\mathcal{I}^{v: \varrho} \mathrm{u}\right)(x) & =\frac{1}{\Gamma(v)} \int_{c_{1}}^{x} \varrho^{\prime}(\kappa)(\varrho(x)-\varrho(\kappa))^{v-1} \mathrm{u}(\kappa) d \kappa, \\
\left(\mathcal{I}_{c_{2}-}^{v: \varrho} \mathrm{u}\right)(x) & =\frac{1}{\Gamma(v)} \int_{x}^{c_{2}} \varrho^{\prime}(\kappa)(\varrho(\kappa)-\varrho(x))^{v-1} \mathrm{u}(\kappa) d \kappa, \quad v>0 .
\end{aligned}
$$


In this article, we will investigate the midpoint version of (9) and some related HHF inequalities by using the weighted fractional integrals (10) with positive weighted symmetric functions in the kernel.

The rest of our article is structured in the following way: In Section 2, we will prove the necessary and auxiliary lemmas, including the midpoint version of (9). In Section 3, we will prove our main results, including new midpoint fractional HHF integral inequalities with some related results. We will present some concluding remarks in Section 4.

\section{Auxiliary Results}

In this section, we prove analogues of the fractional HH inequalities (2)-(3) and HHF inequalities (7)-(8) for weighted fractional integral operators with positive weighted symmetric function kernels. Here, the main results are as follows: Theorem 1 (it is a generalisation of HH inequalities (2)-(3) and HHF inequality (7), and a reformulation of HHF inequality (8)) and Lemma 2 (it is a consequence of Theorem 1).

At first, we need the following lemma.

Lemma 1. Assume that $w:\left[c_{1}, c_{2}\right] \rightarrow(0, \infty)$ is an integrable function and symmetric with respect to $\left(c_{1}+c_{2}\right) / 2, c_{1}<c_{2}$. Then,

(i) for each $\kappa \in[0,1]$, we have

$$
w\left(\frac{\kappa}{2} c_{1}+\frac{2-\kappa}{2} c_{2}\right)=w\left(\frac{2-\kappa}{2} c_{1}+\frac{\kappa}{2} c_{2}\right) .
$$

(ii) For $v>0$, we have

$$
\begin{aligned}
& \left(\varrho^{-1}\left(\frac{c_{1}+c_{2}}{2}\right)+\mathcal{I}^{v: \varrho}(w \circ \varrho)\right)\left(\varrho^{-1}\left(\boldsymbol{c}_{2}\right)\right)=\left(\mathcal{I}_{\varrho^{-1}\left(\frac{c_{1}+c_{2}}{2}\right)-}^{v(w \circ \varrho)}\right)\left(\varrho^{-1}\left(\boldsymbol{c}_{1}\right)\right) \\
& =\frac{1}{2}\left[\left(\varrho^{-1}\left(\frac{\boldsymbol{c}_{1}+c_{2}}{2}\right)+\mathcal{I}^{v: \varrho}(w \circ \varrho)\right)\left(\varrho^{-1}\left(\boldsymbol{c}_{2}\right)\right)+\left(\mathcal{I}_{\varrho^{-1}\left(\frac{c_{1}+c_{2}}{2}\right)-}^{v: \varrho}(w \circ \varrho)\right)\left(\varrho^{-1}\left(\boldsymbol{c}_{1}\right)\right)\right] .
\end{aligned}
$$

Proof.

(i) Let $x=\frac{\kappa}{2} \mathbf{c}_{1}+\frac{2-\kappa}{2} \mathbf{c}_{2}$. It is clear that $x \in\left[\mathbf{c}_{1}, \mathbf{c}_{2}\right]$ for each $\kappa \in[0,1]$ and that $\mathbf{c}_{1}+\mathbf{c}_{2}-x=$ $\frac{2-\kappa}{2} \mathbf{c}_{1}+\frac{\kappa}{2} \mathbf{c}_{2}$. Then, by making use of the assumptions and Definition 1 , we can obtain (12).

(ii) The symmetry property of $w$ leads to

$$
(w \circ \varrho)(\kappa)=w(\varrho(\kappa))=w\left(\mathbf{c}_{1}+\mathbf{c}_{2}-\varrho(\kappa)\right), \quad \forall \kappa \in\left[\varrho^{-1}\left(\mathbf{c}_{1}\right), \varrho^{-1}\left(\mathbf{c}_{2}\right)\right] .
$$

From above and setting $\varrho(x):=\mathbf{c}_{1}+\mathbf{c}_{2}-\varrho(\kappa)$, it follows that

$$
\begin{aligned}
& \left(\varrho^{-1}\left(\frac{\mathbf{c}_{1}+\mathbf{c}_{2}}{2}\right)+\mathcal{I}^{v: \varrho}(w \circ \varrho)\right)\left(\varrho^{-1}\left(\mathbf{c}_{2}\right)\right) \\
& =\frac{1}{\Gamma(v)} \int_{\varrho^{-1}\left(\frac{\mathbf{c}_{1}+\mathbf{c}_{2}}{2}\right)}^{\varrho^{-1}\left(\mathbf{c}_{2}\right)}\left(\mathbf{c}_{2}-\varrho(x)\right)^{v-1}(w \circ \varrho)(x) \varrho^{\prime}(x) d x \\
& =\frac{1}{\Gamma(v)} \int_{\varrho^{-1}\left(\mathbf{c}_{1}\right)}^{\varrho^{-1}\left(\frac{\mathbf{c}_{1}+\mathbf{c}_{2}}{2}\right)}\left(\varrho(\kappa)-\mathbf{c}_{1}\right)^{v-1} w\left(\mathbf{c}_{1}+\mathbf{c}_{2}-\varrho(\kappa)\right) \varrho^{\prime}(\kappa) d \kappa \\
& =\frac{1}{\Gamma(v)} \int_{\varrho^{-1}\left(\mathbf{c}_{1}\right)}^{\varrho^{-1}\left(\frac{\mathbf{c}_{1}+\mathbf{c}_{2}}{2}\right)}\left(\varrho(\kappa)-\mathbf{c}_{1}\right)^{v-1}(w \circ \varrho)(\kappa) \varrho^{\prime}(\kappa) d \kappa \\
& =\left(\mathcal { I } ^ { v : \varrho } \left(\varrho^{-1}\left(\frac{\mathbf{c}_{1}+\mathbf{c}_{2}}{2}\right)-\right.\right.
\end{aligned}
$$


which completes the desired equality (13).

Remark 2. Throughout the present article, we denote $[w(x)]^{-1}=\frac{1}{w(x)}$ and $\varrho^{-1}(x)$ the inverse of the function $\varrho(x)$.

Theorem 1. Let $0 \leq c_{1}<c_{2}$, let $\mathrm{u}:\left[c_{1}, c_{2}\right] \rightarrow \mathcal{R}$ be an $L^{1}$ convex function and $w:\left[c_{1}, c_{2}\right] \rightarrow \mathcal{R}$ be an integrable, positive and weighted symmetric function with respect to $\frac{c_{1}+c_{2}}{2}$. If, in addition, $\varrho$ is an increasing and positive function from $\left[c_{1}, c_{2}\right)$ onto itself such that its derivative $\varrho^{\prime}(x)$ is continuous on $\left(\boldsymbol{c}_{1}, \boldsymbol{c}_{2}\right)$, then for $v>0$, the following inequalities are valid:

$$
\begin{aligned}
& \mathrm{u}\left(\frac{\boldsymbol{c}_{1}+\boldsymbol{c}_{2}}{2}\right)\left[\left(\varrho^{-1}\left(\frac{\boldsymbol{c}_{1}+\boldsymbol{c}_{2}}{2}\right)+\mathcal{I}^{v: \varrho}(w \circ \varrho)\right)\left(\varrho^{-1}\left(\boldsymbol{c}_{2}\right)\right)\right. \\
& \left.+\left(\mathcal{I}_{\varrho^{-1}\left(\frac{\boldsymbol{c}_{1}+\boldsymbol{c}_{2}}{2}\right)-}^{v(w \circ \varrho)}\right)\left(\varrho^{-1}\left(\boldsymbol{c}_{1}\right)\right)\right] \leq w\left(\boldsymbol{c}_{2}\right)\left(\varrho^{-1}\left(\frac{\boldsymbol{c}_{1}+\boldsymbol{c}_{2}}{2}\right)+\mathcal{I}_{w \circ \varrho}^{v: \varrho}(\mathbf{u} \circ \varrho)\right)\left(\varrho^{-1}\left(\boldsymbol{c}_{2}\right)\right) \\
& +w\left(\boldsymbol{c}_{1}\right)\left(w \circ \mathcal{I}_{\varrho^{-1}\left(\frac{\boldsymbol{c}_{1}+\boldsymbol{c}_{2}}{2}\right)-}(\mathbf{u} \circ \varrho)\right)\left(\varrho^{-1}\left(\boldsymbol{c}_{1}\right)\right) \\
& \leq \frac{\mathrm{u}\left(\boldsymbol{c}_{1}\right)+\mathrm{u}\left(\boldsymbol{c}_{2}\right)}{2}\left[\left(\varrho^{-1}\left(\frac{\boldsymbol{c}_{1}+\boldsymbol{c}_{2}}{2}\right)+{ }^{\mathcal{I}: \varrho}(w \circ \varrho)\right)\left(\varrho^{-1}\left(\boldsymbol{c}_{2}\right)\right)\right. \\
& \left.+\left(\mathcal{I}_{\varrho^{-1}\left(\frac{c_{1}+c_{2}}{2}\right)-}(w \circ \varrho)\right)\left(\varrho^{-1}\left(c_{1}\right)\right)\right] .
\end{aligned}
$$

Proof. The convexity of $u$ on $\left[\mathbf{c}_{1}, \mathbf{c}_{2}\right]$ gives

$$
\mathrm{u}\left(\frac{x+y}{2}\right) \leq \frac{\mathrm{u}(x)+\mathrm{u}(y)}{2} \text { for all } x, y \in\left[\mathbf{c}_{1}, \mathbf{c}_{2}\right] .
$$

So, for $x=\frac{\kappa}{2} \mathbf{c}_{1}+\frac{2-\kappa}{2} \mathbf{c}_{2}$ and $y=\frac{2-\kappa}{2} \mathbf{c}_{1}+\frac{\kappa}{2} \mathbf{c}_{2}, \kappa \in[0,1]$, it follows that

$$
2 \mathrm{u}\left(\frac{\mathbf{c}_{1}+\mathbf{c}_{2}}{2}\right) \leq \mathrm{u}\left(\frac{\kappa}{2} \mathrm{c}_{1}+\frac{2-\kappa}{2} \mathrm{c}_{2}\right)+\mathrm{u}\left(\frac{2-\kappa}{2} \mathrm{c}_{1}+\frac{\kappa}{2} \mathrm{c}_{2}\right) .
$$

Multiplying both sides of (15) by $\kappa^{\nu-1} w\left(\frac{\kappa}{2} \mathbf{c}_{1}+\frac{2-\kappa}{2} \mathbf{c}_{2}\right)$ and integrating the resulting inequality with respect to $\kappa$ over $[0,1]$,, we obtain

$$
\begin{aligned}
2 \mathrm{u}\left(\frac{\mathbf{c}_{1}+\mathbf{c}_{2}}{2}\right) \int_{0}^{1} \kappa^{\nu-1} w\left(\frac{\kappa}{2} \mathbf{c}_{1}\right. & \left.+\frac{2-\kappa}{2} \mathbf{c}_{2}\right) d \kappa \\
\leq \int_{0}^{1} \kappa^{\nu-1} \mathrm{u} & \left(\frac{\kappa}{2} \mathbf{c}_{1}+\frac{2-\kappa}{2} \mathbf{c}_{2}\right) w\left(\frac{\kappa}{2} \mathbf{c}_{1}+\frac{2-\kappa}{2} \mathbf{c}_{2}\right) d \kappa \\
& +\int_{0}^{1} \kappa^{\nu-1} \mathrm{u}\left(\frac{2-\kappa}{2} \mathbf{c}_{1}+\frac{\kappa}{2} \mathbf{c}_{2}\right) w\left(\frac{\kappa}{2} \mathbf{c}_{1}+\frac{2-\kappa}{2} \mathbf{c}_{2}\right) d \kappa .
\end{aligned}
$$


From the left-hand side of the inequality in (16), we use (13) to obtain

$$
\begin{aligned}
& \frac{2^{v-1} \Gamma(v)}{\left(\mathbf{c}_{2}-\mathbf{c}_{1}\right)^{v}}\left[\left(\varrho^{-1}\left(\frac{\mathbf{c}_{1}+\mathbf{c}_{2}}{2}\right)+\mathcal{I}^{v: \varrho}(w \circ \varrho)\right)\left(\varrho^{-1}\left(\mathbf{c}_{2}\right)\right)+\left(\mathcal{I}_{\varrho^{-1}\left(\frac{\mathbf{c}_{1}+\mathbf{c}_{2}}{2}\right)-}(w \circ \varrho)\right)\left(\varrho^{-1}\left(\mathbf{c}_{1}\right)\right)\right] \\
& =\frac{2^{v} \Gamma(v)}{\left(\mathbf{c}_{2}-\mathbf{c}_{1}\right)^{v}}\left(\varrho^{-1}\left(\frac{\mathbf{c}_{1}+\mathbf{c}_{2}}{2}\right)+\mathcal{I}^{v: \varrho}(w \circ \varrho)\right)\left(\varrho^{-1}\left(\mathbf{c}_{2}\right)\right) \\
& =\frac{2^{v}}{\left(\mathbf{c}_{2}-\mathbf{c}_{1}\right)^{v}} \int_{\varrho^{-1}\left(\frac{\mathbf{c}_{1}+\mathbf{c}_{2}}{2}\right)}^{\varrho^{-1}\left(\mathbf{c}_{2}-\varrho(x)\right)^{v-1}(w \circ \varrho)(x) \varrho^{\prime}(x) d x} \\
& =\int_{\varrho^{-1}\left(\frac{\mathbf{c}_{1}+\mathbf{c}_{2}}{2}\right)}^{\varrho^{-1}\left(\mathbf{c}_{2}\right)}\left(\frac{2\left(\mathbf{c}_{2}-\varrho(x)\right)}{\mathbf{c}_{2}-\mathbf{c}_{1}}\right)^{v-1}(w \circ \varrho)(x) \varrho^{\prime}(x) \frac{2 d x}{\mathbf{c}_{2}-\mathbf{c}_{1}} \\
& =\int_{0}^{1} \kappa^{v-1} w\left(\frac{\kappa}{2} \mathbf{c}_{1}+\frac{2-\kappa}{2} \mathbf{c}_{2}\right) d \kappa, \quad\left[\text { denoting } \kappa:=\frac{2\left(\mathbf{c}_{2}-\varrho(x)\right)}{\mathbf{c}_{2}-\mathbf{c}_{1}}\right] .
\end{aligned}
$$

It follows that

$$
\begin{aligned}
& 2 \mathrm{u}\left(\frac{\mathbf{c}_{1}+\mathbf{c}_{2}}{2}\right) \int_{0}^{1} \kappa^{v-1} w\left(\frac{\kappa}{2} \mathbf{c}_{1}+\frac{2-\kappa}{2} \mathbf{c}_{2}\right) d \kappa=\frac{2^{v} \Gamma(v)}{\left(\mathbf{c}_{2}-\mathbf{c}_{1}\right)^{v}} \mathrm{u}\left(\frac{\mathbf{c}_{1}+\mathbf{c}_{2}}{2}\right) \\
& \quad \times\left[\left(\varrho^{-1}\left(\frac{\mathbf{c}_{1}+\mathbf{c}_{2}}{2}\right)+\mathcal{I}^{v: \varrho}(w \circ \varrho)\right)\left(\varrho^{-1}\left(\mathbf{c}_{2}\right)\right)+\left(\mathcal{I}_{\varrho^{v:}\left(\frac{\mathbf{c}_{1}+\mathbf{c}_{2}}{2}\right)-}(w \circ \varrho)\right)\left(\varrho^{-1}\left(\mathbf{c}_{1}\right)\right)\right] .
\end{aligned}
$$

By evaluating the weighted fractional operators, we see that

$$
\begin{aligned}
& w\left(\mathbf{c}_{2}\right)\left(\varrho^{-1}\left(\frac{\mathbf{c}_{1}+\mathbf{c}_{2}}{2}\right)+\mathcal{I}_{w \circ \varrho \varrho}^{v: \varrho}(\mathbf{u} \circ \varrho)\right)\left(\varrho^{-1}\left(\mathbf{c}_{2}\right)\right)+w\left(\mathbf{c}_{1}\right)\left(w_{w \circ \varrho} \mathcal{I}_{\varrho^{-1}\left(\frac{\mathbf{c}_{1}+\mathbf{c}_{2}}{2}\right)-}(\mathbf{u} \circ \varrho)\right)\left(\varrho^{-1}\left(\mathbf{c}_{1}\right)\right) \\
& =w\left(\mathbf{c}_{2}\right) \frac{(w \circ \varrho)^{-1}\left(\varrho^{-1}\left(\mathbf{c}_{2}\right)\right)}{\Gamma(v)} \int_{\varrho^{-1}\left(\frac{\mathbf{c}_{1}+\mathbf{c}_{2}}{2}\right)}^{\varrho^{-1}\left(\mathbf{c}_{2}\right)}\left(\mathbf{c}_{2}-\varrho(x)\right)^{v-1}(\mathbf{u} \circ \varrho)(x)(w \circ \varrho)(x) \varrho^{\prime}(x) d x \\
& +w\left(\mathbf{c}_{1}\right) \frac{(w \circ \varrho)^{-1}\left(\varrho^{-1}\left(\mathbf{c}_{1}\right)\right)}{\Gamma(v)} \int_{\varrho^{-1}\left(\mathbf{c}_{1}\right)}^{\varrho^{-1}\left(\frac{\mathbf{c}_{1}+\mathbf{c}_{2}}{2}\right)}\left(\varrho(x)-\mathbf{c}_{1}\right)^{v-1}(\mathbf{u} \circ \varrho)(x)(w \circ \varrho)(x) \varrho^{\prime}(x) d x \\
& =\frac{\left(\mathbf{c}_{2}-\mathbf{c}_{1}\right)^{v}}{2^{v} \Gamma(v)} \int_{\varrho^{-1}\left(\frac{\mathbf{c}_{1}+\mathbf{c}_{2}}{2}\right)}^{\varrho^{-1}\left(\mathbf{c}_{2}\right)}\left(\frac{2\left(\mathbf{c}_{2}-\varrho(x)\right)}{\mathbf{c}_{2}-\mathbf{c}_{1}}\right)^{v-1}(\mathbf{u} \circ \varrho)(x)(w \circ \varrho)(x) \varrho^{\prime}(x) \frac{2 d x}{\mathbf{c}_{2}-\mathbf{c}_{1}} \\
& +\frac{\left(\mathbf{c}_{2}-\mathbf{c}_{1}\right)^{v}}{2^{v} \Gamma(v)} \int_{\varrho^{-1}\left(\mathbf{c}_{1}\right)}^{\varrho^{-1}\left(\frac{\mathbf{c}_{1}+\mathbf{c}_{2}}{2}\right)}\left(\frac{2\left(\varrho(x)-\mathbf{c}_{1}\right)}{\mathbf{c}_{2}-\mathbf{c}_{1}}\right)^{v-1}(\mathbf{u} \circ \varrho)(x)(w \circ \varrho)(x) \varrho^{\prime}(x) \frac{2 d x}{\mathbf{c}_{2}-\mathbf{c}_{1}},
\end{aligned}
$$

where we used

$$
\left[(w \circ \varrho)\left(\varrho^{-1}(y)\right)\right]^{-1}=\frac{1}{(w \circ \varrho)\left(\varrho^{-1}(y)\right)}=\frac{1}{w(y)} \quad \text { for } y=\mathbf{c}_{1}, \mathbf{c}_{2} .
$$


Symmetry 2021, 13, 550

7 of 22

Setting $t_{1}=\frac{2\left(\mathbf{c}_{2}-\varrho(x)\right)}{\mathbf{c}_{2}-\mathbf{c}_{1}}$ and $t_{2}=\frac{2\left(\varrho(x)-\mathbf{c}_{1}\right)}{\mathbf{c}_{2}-\mathbf{c}_{1}}$, one can deduce that

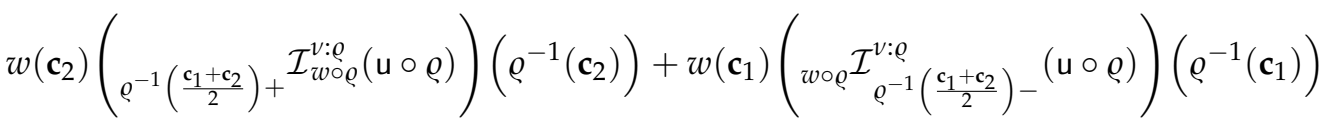

$$
\begin{aligned}
& =\frac{\left(\mathbf{c}_{2}-\mathbf{c}_{1}\right)^{v}}{2^{v} \Gamma(v)}\left[\int_{0}^{1} t_{1}^{v-1} \mathbf{u}\left(\frac{t_{1}}{2} \mathbf{c}_{1}+\frac{2-t_{1}}{2} \mathbf{c}_{2}\right) w\left(\frac{t_{1}}{2} \mathbf{c}_{1}+\frac{2-t_{1}}{2} \mathbf{c}_{2}\right) d t_{1}\right. \\
& +\int_{0}^{1} t_{2}{ }^{v-1} \mathrm{u}\left(\frac{2-t_{2}}{2} \mathbf{c}_{1}+\frac{t_{2}}{2} \mathbf{c}_{2}\right) w\left(\frac{2-t_{2}}{2} \mathbf{c}_{1}+\frac{t_{2}}{2} \mathbf{c}_{2}\right) d t_{2} \\
& =\frac{\left(\mathbf{c}_{2}-\mathbf{c}_{1}\right)^{v}}{2^{v} \Gamma(v)}\left[\int_{0}^{1} \kappa^{v-1} \mathrm{u}\left(\frac{\kappa}{2} \mathbf{c}_{1}+\frac{2-\kappa}{2} \mathbf{c}_{2}\right) w\left(\frac{\kappa}{2} \mathbf{c}_{1}+\frac{2-\kappa}{2} \mathbf{c}_{2}\right) d \kappa\right. \\
& +\int_{0}^{1} \kappa^{\nu-1} \mathrm{u}\left(\frac{2-\kappa}{2} \mathbf{c}_{1}+\frac{\kappa}{2} \mathbf{c}_{2}\right) \underbrace{w\left(\frac{\kappa}{2} \mathbf{c}_{1}+\frac{2-\kappa}{2} \mathbf{c}_{2}\right)}_{\text {by using (12) }} d \kappa] \text {. }
\end{aligned}
$$

It follows that

$$
\begin{aligned}
\int_{0}^{1} \kappa^{v-1} \mathbf{u}\left(\frac{\kappa}{2} \mathbf{c}_{1}+\frac{2-\kappa}{2} \mathbf{c}_{2}\right) w\left(\frac{\kappa}{2} \mathbf{c}_{1}\right. & \left.+\frac{2-\kappa}{2} \mathbf{c}_{2}\right) d \kappa \\
& +\int_{0}^{1} \kappa^{v-1} \mathbf{u}\left(\frac{2-\kappa}{2} \mathbf{c}_{1}+\frac{\kappa}{2} \mathbf{c}_{2}\right) w\left(\frac{\kappa}{2} \mathbf{c}_{1}+\frac{2-\kappa}{2} \mathbf{c}_{2}\right) d \kappa \\
=\frac{2^{v} \Gamma(v)}{\left(\mathbf{c}_{2}-\mathbf{c}_{1}\right)^{v}}\left[w ( \mathbf { c } _ { 2 } ) \left(\varrho^{-1}\left(\frac{\mathbf{c}_{1}+\mathbf{c}_{2}}{2}\right)+\right.\right. & \left.\mathcal{I}_{w \circ \varrho}^{v: \varrho}(\mathbf{u} \circ \varrho)\right)\left(\varrho^{-1}\left(\mathbf{c}_{2}\right)\right) \\
& \left.+w\left(\mathbf{c}_{1}\right)\left(w \circ \mathcal{I}_{\varrho^{-1}\left(\frac{\mathbf{c}_{1}+\mathbf{c}_{2}}{2}\right)-}(\mathbf{u} \circ \varrho)\right)\left(\varrho^{-1}\left(\mathbf{c}_{1}\right)\right)\right]
\end{aligned}
$$

By making use of (17) and (19) in (16), we get

$$
\begin{aligned}
& \mathrm{u}\left(\frac{\mathbf{c}_{1}+\mathbf{c}_{2}}{2}\right)\left[\left(\varrho^{-1}\left(\frac{\mathbf{c}_{1}+\mathbf{c}_{2}}{2}\right)+\mathcal{I}^{v: \varrho}(w \circ \varrho)\right)\left(\varrho^{-1}\left(\mathbf{c}_{2}\right)\right)\right.
\end{aligned}
$$

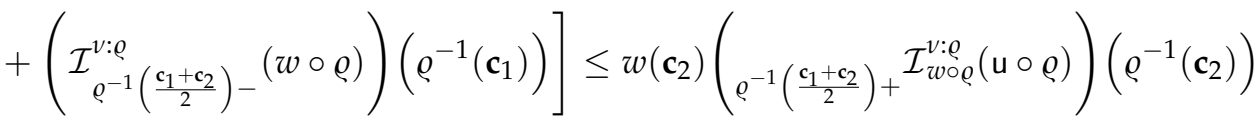

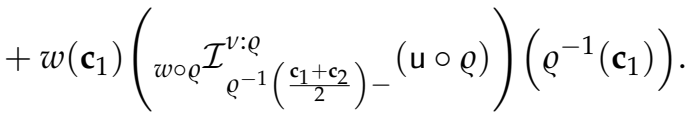

Thus, the proof of the first inequality of (14) is completed.

On the other hand, we can prove the second inequality of (14) by making use of the convexity of $u$ to get

$$
\mathrm{u}\left(\frac{\kappa}{2} \mathrm{c}_{1}+\frac{2-\kappa}{2} \mathrm{c}_{2}\right)+\mathrm{u}\left(\frac{2-\kappa}{2} \mathrm{c}_{1}+\frac{\kappa}{2} \mathrm{c}_{2}\right) \leq \mathrm{u}\left(\mathrm{c}_{1}\right)+\mathrm{u}\left(\mathrm{c}_{2}\right) .
$$


Multiplying both sides of (21) by $\kappa^{\nu-1} w\left(\frac{\kappa}{2} \mathbf{c}_{1}+\frac{2-\kappa}{2} \mathbf{c}_{2}\right)$ and integrating with respect to $\kappa$ over $[0,1]$ to get

$$
\begin{aligned}
& \int_{0}^{1} \kappa^{\nu-1} \mathrm{u}\left(\frac{\kappa}{2} \mathbf{c}_{1}+\right.\left.\frac{2-\kappa}{2} \mathbf{c}_{2}\right) w\left(\frac{\kappa}{2} \mathbf{c}_{1}+\frac{2-\kappa}{2} \mathbf{c}_{2}\right) d \kappa \\
&+\int_{0}^{1} \kappa^{\nu-1} \mathrm{u}\left(\frac{2-\kappa}{2} \mathbf{c}_{1}+\frac{\kappa}{2} \mathbf{c}_{2}\right) w\left(\frac{\kappa}{2} \mathbf{c}_{1}+\frac{2-\kappa}{2} \mathbf{c}_{2}\right) d \kappa \\
& \quad \leq\left(\mathbf{u}\left(\mathbf{c}_{1}\right)+\mathbf{u}\left(\mathbf{c}_{2}\right)\right) \int_{0}^{1} \kappa^{\nu-1} w\left(\frac{\kappa}{2} \mathbf{c}_{1}+\frac{2-\kappa}{2} \mathbf{c}_{2}\right) d \kappa
\end{aligned}
$$

Then, by using (12) and (19) in (22), we get

$$
\begin{aligned}
w\left(\mathbf{c}_{2}\right)\left(\varrho^{-1}\left(\frac{\mathbf{c}_{1}+\mathbf{c}_{2}}{2}\right)+\right. & \left.\mathcal{I}_{w \circ \varrho}^{v: \varrho}(\mathbf{u} \circ \varrho)\right)\left(\varrho^{-1}\left(\mathbf{c}_{2}\right)\right) \\
& +w\left(\mathbf{c}_{1}\right)\left(w \circ \mathcal{I}_{\varrho^{-1}\left(\frac{\mathbf{c}_{1}+\mathbf{c}_{2}}{2}\right)-}(\mathbf{u} \circ \varrho)\right)\left(\varrho^{-1}\left(\mathbf{c}_{1}\right)\right) \\
\leq & \frac{\mathbf{u}\left(\mathbf{c}_{1}\right)+\mathbf{u}\left(\mathbf{c}_{2}\right)}{2}\left[\left(\varrho^{-1\left(\frac{\mathbf{c}_{1}+\mathbf{c}_{2}}{2}\right)+}+\frac{\left.\mathcal{I}^{v: \varrho}(w \circ \varrho)\right)\left(\varrho^{-1}\left(\mathbf{c}_{2}\right)\right)}{}\right.\right. \\
& \left.+\left(\mathcal{I}_{\varrho^{-1}\left(\frac{\mathbf{c}_{1}+\mathbf{c}_{2}}{2}\right)-}(w \circ \varrho)\right)\left(\varrho^{-1}\left(\mathbf{c}_{1}\right)\right)\right] .
\end{aligned}
$$

This ends our proof.

Remark 3. From Theorem 1, we can obtain some special cases as follows:

(i) If $\varrho(x)=x$, then inequality (14) becomes

$$
\begin{aligned}
& \mathrm{u}\left(\frac{\boldsymbol{c}_{1}+\boldsymbol{c}_{2}}{2}\right)\left[\left(\frac{\boldsymbol{c}_{1}+c_{2}}{2}\right)+{ }^{R L} \mathcal{I}^{v} w\left(\boldsymbol{c}_{2}\right)+{ }^{R L} \mathcal{I}_{\left(\frac{c_{1}+c_{2}}{2}\right)-}^{v} w\left(\boldsymbol{c}_{1}\right)\right]
\end{aligned}
$$

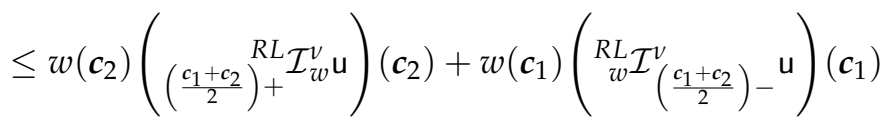

$$
\begin{aligned}
& \leq \frac{\mathrm{u}\left(\boldsymbol{c}_{1}\right)+\mathrm{u}\left(\boldsymbol{c}_{2}\right)}{2}\left[\left(\frac{\boldsymbol{c}_{1}+c_{2}}{2}\right)+{ }^{R L} \mathcal{I}^{v} w\left(\boldsymbol{c}_{2}\right)+{ }^{R L} \mathcal{I}^{v}\left(\frac{\boldsymbol{c}_{1}+c_{2}}{2}\right)-{ } w\left(\boldsymbol{c}_{1}\right)\right],
\end{aligned}
$$

where ${ }_{c_{1}+}^{R L} \mathcal{I}_{w}^{v}$ and ${ }_{w}^{R L} \mathcal{I}_{c_{2}-}^{v}$ are the left- and right-weighted $\mathrm{RL}$ fractional integrals, respectively, given by

$$
\begin{aligned}
& \left({ }_{c_{1}+}^{R L} \mathcal{I}_{w}^{v} \mathrm{u}\right)(x)=\frac{w^{-1}(x)}{\Gamma(v)} \int_{c_{1}}^{x}(x-\kappa)^{v-1} \mathbf{u}(\kappa) w(\kappa) d \kappa, \\
& \left({ }_{w}^{R L} \mathcal{I}_{c_{2}-}^{v} \mathbf{u}\right)(x)=\frac{w^{-1}(x)}{\Gamma(v)} \int_{x}^{c_{2}}(\kappa-x)^{v-1} \mathbf{u}(\kappa) w(\kappa) d \kappa, \quad v>0 .
\end{aligned}
$$

(ii) If $\varrho(x)=x$ and $v=1$, then inequality (14) becomes the inequality in (7).

(iii) If $\varrho(x)=x$ and $w(x)=1$, then inequality (14) becomes the inequality in (5).

(iv) If $\varrho(x)=x, w(x)=1$ and $v=1$, then inequality (14) becomes the inequality in (2).

Lemma 2. Let $0 \leq c_{1}<c_{2}$, let $\mathrm{u}:\left[c_{1}, c_{2}\right] \rightarrow \mathcal{R}$ be a continuous with a derivative $\mathrm{u}^{\prime} \in L^{1}\left[\boldsymbol{c}_{1}, \boldsymbol{c}_{2}\right]$ such that $\mathrm{u}(x)=\mathrm{u}\left(\boldsymbol{c}_{1}\right)+\int_{c_{1}}^{x} \mathrm{u}^{\prime}(\kappa) d \kappa$ and let $w:\left[\boldsymbol{c}_{1}, \boldsymbol{c}_{2}\right] \rightarrow \mathcal{R}$ be an integrable, positive and weighted symmetric function with respect to $\frac{c_{1}+c_{2}}{2}$. If $\varrho$ is a continuous increasing mapping from 
the interval $\left[c_{1}, c_{2}\right)$ onto itself with a derivative $Q^{\prime}(x)$ which is continuous on $\left(\boldsymbol{c}_{1}, \boldsymbol{c}_{2}\right)$, then for $v>0$, the following equality is valid:

$$
\begin{aligned}
& \mathrm{u}\left(\frac{\boldsymbol{c}_{1}+\boldsymbol{c}_{2}}{2}\right)\left[\left(\varrho^{-1}\left(\frac{\boldsymbol{c}_{1}+\boldsymbol{c}_{2}}{2}\right)+\mathcal{I}^{v: \varrho}(w \circ \varrho)\right)\left(\varrho^{-1}\left(\boldsymbol{c}_{2}\right)\right)\right. \\
& \left.+\left(\mathcal{I}_{\varrho^{-1}\left(\frac{\boldsymbol{c}_{1}+\boldsymbol{c}_{2}}{2}\right)-}(w \circ \varrho)\right)\left(\varrho^{-1}\left(\boldsymbol{c}_{1}\right)\right)\right] \\
& -\left[w\left(\boldsymbol{c}_{2}\right)\left(\varrho^{-1}\left(\frac{\boldsymbol{c}_{1}+c_{2}}{2}\right)+\mathcal{I}_{w \circ \varrho}^{v: \varrho}(\mathbf{u} \circ \varrho)\right)\left(\varrho^{-1}\left(\boldsymbol{c}_{2}\right)\right)\right. \\
& \left.+w\left(\boldsymbol{c}_{1}\right)\left(w \circ \mathcal{I}_{\varrho^{-1}}^{v: \varrho}\left(\frac{\boldsymbol{c}_{1}+\boldsymbol{c}_{2}}{2}\right)-(\mathbf{u} \circ \varrho)\right)\left(\varrho^{-1}\left(\boldsymbol{c}_{1}\right)\right)\right] \\
& =\frac{1}{\Gamma(v)} \int_{\varrho^{-1}\left(c_{1}\right)}^{\varrho^{-1}\left(\frac{c_{1}+c_{2}}{2}\right)}\left[\int_{\varrho^{-1}\left(c_{1}\right)}^{\kappa} \varrho^{\prime}(x)\left(\varrho(x)-c_{1}\right)^{\nu-1}(w \circ \varrho)(x) d x\right]\left(\mathbf{u}^{\prime} \circ \varrho\right)(\kappa) \varrho^{\prime}(\kappa) d \kappa \\
& -\frac{1}{\Gamma(v)} \int_{\varrho^{-1}\left(\frac{c_{1}+c_{2}}{2}\right)}^{\varrho^{-1}\left(c_{2}\right)}\left[\int_{\kappa}^{\varrho^{-1}\left(c_{2}\right)} \varrho^{\prime}(x)\left(c_{2}-\varrho(x)\right)^{\nu-1}(w \circ \varrho)(x) d x\right]\left(\mathbf{u}^{\prime} \circ \varrho\right)(\kappa) \varrho^{\prime}(\kappa) d \kappa .
\end{aligned}
$$

Proof. Let us set

$$
\begin{aligned}
& \frac{1}{\Gamma(v)} \int_{\varrho^{-1}\left(\mathbf{c}_{1}\right)}^{\varrho^{-1}\left(\frac{\mathbf{c}_{1}+\mathbf{c}_{2}}{2}\right)}\left[\int_{\varrho^{-1}\left(\mathbf{c}_{1}\right)}^{\kappa} \varrho^{\prime}(x)\left(\varrho(x)-\mathbf{c}_{1}\right)^{v-1}(w \circ \varrho)(x) d x\right]\left(\mathbf{u}^{\prime} \circ \varrho\right)(\kappa) \varrho^{\prime}(\kappa) d \kappa \\
& -\frac{1}{\Gamma(v)} \int_{\varrho^{-1}\left(\frac{\mathbf{c}_{1}+\mathbf{c}_{2}}{2}\right)}^{\varrho^{-1}\left(\mathbf{c}_{2}\right)}\left[\int_{\kappa}^{\varrho^{-1}\left(\mathbf{c}_{2}\right)} \varrho^{\prime}(x)\left(\mathbf{c}_{2}-\varrho(x)\right)^{v-1}(w \circ \varrho)(x) d x\right]\left(\mathbf{u}^{\prime} \circ \varrho\right)(\kappa) \varrho^{\prime}(\kappa) d \kappa \\
& =\frac{1}{\Gamma(v)} \int_{\varrho^{-1}\left(\mathbf{c}_{1}\right)}^{\varrho^{-1}\left(\frac{\mathbf{c}_{1}+\mathbf{c}_{2}}{2}\right)}\left[\int_{\varrho^{-1}\left(\mathbf{c}_{1}\right)}^{\kappa} \varrho^{\prime}(x)\left(\varrho(x)-\mathbf{c}_{1}\right)^{v-1}(w \circ \varrho)(x) d x\right]\left(\mathbf{u}^{\prime} \circ \varrho\right)(\kappa) \varrho^{\prime}(\kappa) d \kappa \\
& +\frac{-1}{\Gamma(v)} \int_{\varrho^{-1}\left(\frac{\mathbf{c}_{1}+\mathbf{c}_{2}}{2}\right)}^{\varrho^{-1}\left(\mathbf{c}_{2}\right)}\left[\int_{\kappa}^{\varrho^{-1}\left(\mathbf{c}_{2}\right)} \varrho^{\prime}(x)\left(\mathbf{c}_{2}-\varrho(x)\right)^{\nu-1}(w \circ \varrho)(x) d x\right]\left(\mathbf{u}^{\prime} \circ \varrho\right)(\kappa) \varrho^{\prime}(\kappa) d \kappa \\
& :=\Xi_{1}+\Xi_{2} .
\end{aligned}
$$

By integrating by parts, using Lemma 1, and (10) and (11), we obtain

$$
\begin{aligned}
\Xi_{1} & =\left.\frac{1}{\Gamma(v)}\left(\int_{\varrho^{-1}\left(\mathbf{c}_{1}\right)}^{\kappa} \varrho^{\prime}(x)\left(\varrho(x)-\mathbf{c}_{1}\right)^{v-1}(w \circ \varrho)(x) d x\right)(\mathbf{u} \circ \varrho)(\kappa) d \kappa\right|_{\kappa=\varrho^{-1}\left(\mathbf{c}_{1}\right)} ^{\varrho^{-1}\left(\frac{\mathbf{c}_{1}+\mathbf{c}_{2}}{2}\right)} \\
& -\frac{1}{\Gamma(v)} \int_{\varrho^{-1}\left(\mathbf{c}_{1}\right)}^{\varrho^{-1}\left(\frac{\mathbf{c}_{1}+\mathbf{c}_{2}}{2}\right)} \varrho^{\prime}(\kappa)\left(\varrho(\kappa)-\mathbf{c}_{1}\right)^{v-1}(w \circ \varrho)(\kappa)(\mathbf{u} \circ \varrho)(\kappa) d \kappa \\
& =\left(\frac{1}{\Gamma(v)} \int_{\varrho^{-1}\left(\mathbf{c}_{1}\right)}^{\varrho^{-1}\left(\frac{\mathbf{c}_{1}+\mathbf{c}_{2}}{2}\right)} \varrho^{\prime}(x)\left(\varrho(x)-\mathbf{c}_{1}\right)^{v-1}(w \circ \varrho)(x) d x\right) \mathbf{u}\left(\frac{\mathbf{c}_{1}+\mathbf{c}_{2}}{2}\right) \\
& -\underbrace{w\left(\mathbf{c}_{1}\right) \frac{(w \circ \varrho)^{-1}\left(\varrho^{-1}\left(\mathbf{c}_{1}\right)\right)}{\Gamma(v)} \int_{\varrho^{-1}\left(\mathbf{c}_{1}\right)}^{\varrho^{-1}\left(\frac{\mathbf{c}_{1}+\mathbf{c}_{2}}{2}\right)} \varrho^{\prime}(\kappa)\left(\varrho(\kappa)-\mathbf{c}_{1}\right)^{v-1}(w \circ \varrho)(\kappa)(\mathbf{u} \circ \varrho)(\kappa) d \kappa}_{\text {by using }(18)}
\end{aligned}
$$




$$
\begin{aligned}
& =\mathrm{u}\left(\frac{\mathbf{c}_{1}+\mathbf{c}_{2}}{2}\right)\left(\mathcal{I}_{\varrho^{v:}\left(\frac{\mathbf{c}_{1}+\mathbf{c}_{2}}{2}\right)-}^{v:}(w \circ \varrho)\right)\left(\varrho^{-1}\left(\mathbf{c}_{1}\right)\right) \\
& -w\left(\mathbf{c}_{1}\right)\left(w \circ \varrho \mathcal{I}^{v: \varrho} \varrho^{-1}\left(\frac{\mathbf{c}_{1}+\mathbf{c}_{2}}{2}\right)-(\mathbf{u} \circ \varrho)\right)\left(\varrho^{-1}\left(\mathbf{c}_{1}\right)\right) \\
& =\frac{1}{2} \mathrm{u}\left(\frac{\mathbf{c}_{1}+\mathbf{c}_{2}}{2}\right)\left[\left(\varrho^{-1}\left(\frac{\mathbf{c}_{1}+\mathbf{c}_{2}}{2}\right)+{ }^{v: \varrho}(w \circ \varrho)\right)\left(\varrho^{-1}\left(\mathbf{c}_{2}\right)\right)\right. \\
& \left.+\left(\mathcal{I}_{\varrho^{-1}\left(\frac{\mathbf{c}_{1}+\mathbf{c}_{2}}{2}\right)-}(w \circ \varrho)\right)\left(\varrho^{-1}\left(\mathbf{c}_{1}\right)\right)\right]-w\left(\mathbf{c}_{1}\right)\left({ }_{w \circ \varrho} \mathcal{I}_{\varrho^{v:}\left(\frac{\mathbf{c}_{1}+\mathbf{c}_{2}}{2}\right)-}(\mathbf{u} \circ \varrho)\right)\left(\varrho^{-1}\left(\mathbf{c}_{1}\right)\right) .
\end{aligned}
$$

Analogously, we get

$$
\begin{aligned}
& \Xi_{2}=\frac{-1}{\Gamma(v)}\left(\int_{\kappa}^{\varrho^{-1}\left(\mathbf{c}_{2}\right)} \varrho^{\prime}(x)\left(\mathbf{c}_{2}-\varrho(x)\right)^{v-1}(w \circ \varrho)(x) d x\right)(\mathrm{u} \circ \varrho)(\kappa) d \kappa||_{t=\varrho^{-1}\left(\frac{\mathbf{c}_{1}+\mathbf{c}_{2}}{2}\right)}^{\varrho^{-1}\left(\mathbf{c}_{2}\right)} \\
& -\frac{1}{\Gamma(v)} \int_{\varrho^{-1}\left(\frac{\mathbf{c}_{1}+\mathbf{c}_{2}}{2}\right)}^{\varrho^{-1}\left(\mathbf{c}_{2}\right)} \varrho^{\prime}(\kappa)\left(\mathbf{c}_{2}-\varrho(\kappa)\right)^{\nu-1}(w \circ \varrho)(\kappa)(\mathbf{u} \circ \varrho)(\kappa) d \kappa \\
& =\left(\frac{1}{\Gamma(v)} \int_{\varrho^{-1}\left(\frac{\mathfrak{c}_{1}+\mathbf{c}_{2}}{2}\right)}^{\varrho^{-1}\left(\mathbf{c}_{2}\right)} \varrho^{\prime}(x)\left(\mathbf{c}_{2}-\varrho(x)\right)^{v-1}(w \circ \varrho)(x) d x\right) \mathrm{u}\left(\frac{\mathbf{c}_{1}+\mathbf{c}_{2}}{2}\right) \\
& -\underbrace{w\left(\mathbf{c}_{2}\right) \frac{(w \circ \varrho)^{-1}\left(\varrho^{-1}\left(\mathbf{c}_{2}\right)\right)}{\Gamma(v)}}_{\text {by using }(18)} \int_{\varrho^{-1}\left(\frac{\mathbf{c}_{1}+\mathbf{c}_{2}}{2}\right)}^{\varrho^{-1}\left(\mathbf{c}_{2}\right)} \varrho^{\prime}(\kappa)\left(\mathbf{c}_{2}-\varrho(\kappa)\right)^{v-1}(w \circ \varrho)(\kappa)(\mathbf{u} \circ \varrho)(\kappa) d \kappa \\
& =\mathrm{u}\left(\frac{\mathbf{c}_{1}+\mathbf{c}_{2}}{2}\right)\left(\varrho^{-1}\left(\frac{\mathbf{c}_{1}+\mathbf{c}_{2}}{2}\right)+\mathcal{I}^{v: \varrho}(w \circ \varrho)\right)\left(\varrho^{-1}\left(\mathbf{c}_{2}\right)\right) \\
& -w\left(\mathbf{c}_{2}\right)\left(\varrho^{-1}\left(\frac{\mathbf{c}_{1}+\mathbf{c}_{2}}{2}\right)+\mathcal{I}_{w \circ \varrho}^{v: \varrho}(\mathbf{u} \circ \varrho)\right)\left(\varrho^{-1}\left(\mathbf{c}_{2}\right)\right) \\
& =\frac{1}{2} \mathrm{u}\left(\frac{\mathbf{c}_{1}+\mathbf{c}_{2}}{2}\right)\left[\left(\varrho^{-1}\left(\frac{\mathbf{c}_{1}+\mathbf{c}_{2}}{2}\right)+\mathcal{I}^{v: \varrho}(w \circ \varrho)\right)\left(\varrho^{-1}\left(\mathbf{c}_{2}\right)\right)\right. \\
& \left.+\left(\mathcal{I}_{\varrho^{-1}\left(\mathbf{c}_{2}\right)-}^{v: \varrho}(w \circ \varrho)\right)\left(\varrho^{-1}\left(\mathbf{c}_{1}\right)\right)\right]-w\left(\mathbf{c}_{2}\right)\left(\varrho^{-1}\left(\frac{\mathbf{c}_{1}+\mathbf{c}_{2}}{2}\right)+\mathcal{I}_{w \circ \varrho}^{v: \varrho}(\mathbf{u} \circ \varrho)\right)\left(\varrho^{-1}\left(\mathbf{c}_{2}\right)\right) \text {. }
\end{aligned}
$$

Thus, we deduce:

$$
\begin{aligned}
\Xi_{1}+\Xi_{2}=\mathrm{u}\left(\frac{\mathbf{c}_{1}+\mathbf{c}_{2}}{2}\right)\left[\left(\varrho^{-1}\left(\frac{\mathbf{c}_{1}+\mathbf{c}_{2}}{2}\right)+\right.\right. & \left.\mathcal{I}^{v: \varrho}(w \circ \varrho)\right)\left(\varrho^{-1}\left(\mathbf{c}_{2}\right)\right) \\
\left.+\left(\underset{\mathcal{I}^{v: \varrho}}{\varrho^{-1}\left(\frac{\mathbf{c}_{1}+\mathbf{c}_{2}}{2}\right)-}(w \circ \varrho)\right)\left(\varrho^{-1}\left(\mathbf{c}_{1}\right)\right)\right] & -\left[w\left(\mathbf{c}_{2}\right)\left(\varrho^{-1}\left(\frac{\mathbf{c}_{1}+\mathbf{c}_{2}}{2}\right)+\mathcal{I}_{w \circ \varrho}^{v: \varrho}(\mathbf{u} \circ \varrho)\right)\left(\varrho^{-1}\left(\mathbf{c}_{2}\right)\right)\right. \\
& \left.+w\left(\mathbf{c}_{1}\right)\left({ }_{w \circ \varrho} \mathcal{I}_{\varrho^{v: \varrho}\left(\frac{\mathbf{c}_{1}+\mathbf{c}_{2}}{2}\right)-}(\mathbf{u} \circ \varrho)\right)\left(\varrho^{-1}\left(\mathbf{c}_{1}\right)\right)\right],
\end{aligned}
$$

which completes the proof of Lemma 2.

Remark 4. From Lemma 2, we can obtain some special cases as follows: 
(i) If $\varrho(x)=x$, then equality (24) becomes

$$
\begin{gathered}
\mathrm{u}\left(\frac{\boldsymbol{c}_{1}+\boldsymbol{c}_{2}}{2}\right)\left[\left(\frac{c_{1}+c_{2}}{2}\right)+{ }^{R L} \mathcal{I}^{v} w\left(\boldsymbol{c}_{2}\right)+{ }^{R L} \mathcal{I}_{\left(\frac{c_{1}+c_{2}}{2}\right)-}^{v} w\left(\boldsymbol{c}_{1}\right)\right] \\
\left.-\left[w\left(c_{2}\right)\left(\frac{c_{1}+c_{2}}{2}\right)+{ }^{R L} \mathcal{I}_{w}^{v} \mathrm{u}\right)\left(c_{2}\right)+w\left(c_{1}\right)\left({ }^{R L} \mathcal{I}^{v}\left(\frac{c_{1}+c_{2}}{2}\right)-\mathrm{u}\right)\left(c_{1}\right)\right] \\
=\frac{1}{\Gamma(v)} \int_{c_{1}}^{\frac{c_{1}+c_{2}}{2}}\left[\int_{c_{1}}^{\mathcal{K}}\left(x-c_{1}\right)^{v-1} w(x) d x\right] \mathrm{u}^{\prime}(\kappa) d \kappa \\
-\frac{1}{\Gamma(v)} \int_{\frac{c_{1}+c_{2}}{2}}^{c_{c_{2}}}\left[\int_{\kappa}^{c_{2}}\left(c_{2}-x\right)^{v-1} w(x) d x\right] \mathrm{u}^{\prime}(\kappa) d \kappa,
\end{gathered}
$$

where $\left(\frac{c_{1}+c_{2}}{2}\right)^{R L}+\mathcal{I}_{w}^{v}$ and ${ }_{w}^{R L} \mathcal{I}^{v}\left(\frac{c_{1}+c_{2}}{2}\right)-$ are as defined in Remark 3.

(ii) If $\varrho(x)=x$ and $w(x)=1$, then equality (24) becomes

$$
\begin{aligned}
& \frac{2^{v-1} \Gamma(v+1)}{\left(c_{2}-c_{1}\right)^{v}}\left[\left(\frac{c_{1}+c_{2}}{2}\right)^{R L}+\mathcal{I}^{v} \mathrm{u}\left(\boldsymbol{c}_{2}\right)+{ }^{R L} \mathcal{I}^{v}\left(\frac{c_{1}+c_{2}}{2}\right)-{ }^{v}\left(c_{1}\right)\right]-\mathrm{u}\left(\frac{c_{1}+c_{2}}{2}\right)=\frac{c_{2}-c_{1}}{4} \\
& \times\left[\int_{0}^{1} \kappa^{v} \mathrm{u}^{\prime}\left(\frac{\kappa}{2} c_{1}+\frac{2-\kappa}{2} c_{2}\right) d \kappa-\int_{0}^{1} \kappa^{v} \mathrm{u}^{\prime}\left(\frac{2-\kappa}{2} c_{1}+\frac{\kappa}{2} c_{2}\right) d \kappa\right],
\end{aligned}
$$

which is already obtained in ([39] [Lemma 3]).

(iii) If $\varrho(x)=x, w(x)=1$ and $v=1$, then equality (24) becomes

$$
\begin{aligned}
\frac{1}{c_{2}-c_{1}} \int_{c_{1}}^{c_{2}} \mathrm{u}(x) d x-\mathrm{u}\left(\frac{c_{1}+c_{2}}{2}\right)=\frac{c_{2}-c_{1}}{4} & {\left[\int_{0}^{1} \kappa \mathrm{u}^{\prime}\left(\frac{\kappa}{2} c_{1}+\frac{2-\kappa}{2} c_{2}\right) d \kappa\right.} \\
& \left.-\int_{0}^{1} \kappa \mathrm{u}^{\prime}\left(\frac{2-\kappa}{2} c_{1}+\frac{\kappa}{2} c_{2}\right) d \kappa\right],
\end{aligned}
$$

which is already obtained in ([39] [Corollary 1]).

\section{Main Results}

By the help of Lemma 2, we can deduce the following HHF inequalities.

Theorem 2. Let $0 \leq c_{1}<c_{2}$, let $\mathrm{u}:\left[c_{1}, c_{2}\right] \subseteq[0, \infty) \rightarrow \mathcal{R}$ be a (continuously) differentiable function on the interval $\left[c_{1}, c_{2}\right]$ such that $\mathrm{u}(x)=\mathrm{u}\left(c_{1}\right)+\int_{c_{1}}^{x} \mathrm{u}^{\prime}(\kappa) d \kappa$, and let $w:\left[c_{1}, c_{2}\right] \rightarrow \mathcal{R}$ be an integrable, positive and weighted symmetric function with respect to $\frac{c_{1}+c_{2}}{2}$. If, in addition, $\left|\mathrm{u}^{\prime}\right|$ is convex on $\left[\boldsymbol{c}_{1}, \boldsymbol{c}_{2}\right]$, and $\varrho$ is an increasing and positive function from $\left[\boldsymbol{c}_{1}, \boldsymbol{c}_{2}\right)$ onto itself such that its derivative $Q^{\prime}(x)$ is continuous on $\left(c_{1}, c_{2}\right)$, then for $v>0$ the following inequalities are valid:

$$
\begin{aligned}
\left|\Xi_{1}+\Xi_{2}\right|=\mid \frac{1}{\Gamma(v)} \int_{\varrho^{-1}\left(c_{1}\right)}^{\varrho^{-1}\left(\frac{c_{1}+c_{2}}{2}\right)}\left[\int_{\varrho^{-1}\left(c_{1}\right)}^{\kappa} \varrho^{\prime}(x)\left(\varrho(x)-c_{1}\right)^{v-1}(w \circ \varrho)(x) d x\right] \\
\quad \times\left(\mathbf{u}^{\prime} \circ \varrho\right)(\kappa) \varrho^{\prime}(\kappa) d \kappa
\end{aligned}
$$




$$
\begin{gathered}
\leq \frac{\left(c_{2}-c_{1}\right)^{v+1}}{2^{v+2} \Gamma(v+3)}\left\{\|w\|_{\left[c_{1}, \frac{c_{1}+c_{2}}{2}\right], \infty}\left[(v+3)\left|\mathbf{u}^{\prime}\left(c_{1}\right)\right|+(v+1)\left|\mathbf{u}^{\prime}\left(c_{2}\right)\right|\right]\right. \\
\left.+\|w\|_{\left[\frac{c_{1}+c_{2}}{2}, c_{2}\right], \infty}\left[(v+1)\left|\mathbf{u}^{\prime}\left(c_{1}\right)\right|+(v+3)\left|\mathbf{u}^{\prime}\left(c_{2}\right)\right|\right]\right\} \\
\leq \frac{\left(c_{2}-c_{1}\right)^{v+1}\|w\|_{\left[c_{1}, c_{2}\right], \infty}\left[\left|\mathbf{u}^{\prime}\left(c_{1}\right)\right|+\left|\mathbf{u}^{\prime}\left(c_{2}\right)\right|\right] .}{2^{v+1} \Gamma(v+2)}
\end{gathered}
$$

Proof. By making use of Lemma 2 and properties of the modulus, we obtain

$$
\begin{aligned}
& \left|\Xi_{1}+\Xi_{2}\right| \\
& =\mid \frac{1}{\Gamma(v)} \int_{\varrho^{-1}\left(\mathbf{c}_{1}\right)}^{\varrho^{-1}\left(\frac{\mathbf{c}_{1}+\mathbf{c}_{2}}{2}\right)}\left[\int_{\varrho^{-1}\left(\mathbf{c}_{1}\right)}^{\kappa} \varrho^{\prime}(x)\left(\varrho(x)-\mathbf{c}_{1}\right)^{v-1}(w \circ \varrho)(x) d x\right]\left(\mathbf{u}^{\prime} \circ \varrho\right)(\kappa) \varrho^{\prime}(\kappa) d \kappa \\
& -\frac{1}{\Gamma(v)} \int_{\varrho^{-1}\left(\frac{\mathbf{c}_{1}+\mathbf{c}_{2}}{2}\right)}^{\varrho^{-1}\left(\mathbf{c}_{2}\right)}\left[\int_{\mathcal{\kappa}}^{\varrho^{-1}\left(\mathbf{c}_{2}\right)} \varrho^{\prime}(x)\left(\mathbf{c}_{2}-\varrho(x)\right)^{v-1}(w \circ \varrho)(x) d x\right]\left(\mathbf{u}^{\prime} \circ \varrho\right)(\kappa) \varrho^{\prime}(\kappa) d \kappa \mid \\
& \leq \frac{1}{\Gamma(v)} \int_{\varrho^{-1}\left(\mathbf{c}_{1}\right)}^{\varrho^{-1}\left(\frac{\mathbf{c}_{1}+\mathbf{c}_{2}}{2}\right)}\left|\int_{\varrho^{-1}\left(\mathbf{c}_{1}\right)}^{\kappa} \varrho^{\prime}(x)\left(\varrho(x)-\mathbf{c}_{1}\right)^{v-1}(w \circ \varrho)(x) d x\right|\left|\left(\mathbf{u}^{\prime} \circ \varrho\right)(\kappa)\right| \varrho^{\prime}(\kappa) d \kappa \\
& \quad+\frac{1}{\Gamma(v)} \int_{\varrho^{-1}\left(\frac{\mathbf{c}_{1}+\mathbf{c}_{2}}{2}\right)}^{\varrho^{-1}\left(\mathbf{c}_{2}\right)}\left|\int_{\mathcal{K}}^{\varrho^{-1}\left(\mathbf{c}_{2}\right)} \varrho^{\prime}(x)\left(\mathbf{c}_{2}-\varrho(x)\right)^{v-1}(w \circ \varrho)(x) d x\right| \\
& \times\left|\left(\mathbf{u}^{\prime} \circ \varrho\right)(\kappa)\right| \varrho^{\prime}(\kappa) d \kappa .
\end{aligned}
$$

Since $\left|\mathbf{u}^{\prime}\right|$ is convex on $\left[\mathbf{c}_{1}, \mathbf{c}_{2}\right]$, we get for $\kappa \in\left[\varrho^{-1}\left(\mathbf{c}_{1}\right), \varrho^{-1}\left(\mathbf{c}_{2}\right)\right]$ :

$$
\begin{aligned}
\left|\left(\mathbf{u}^{\prime} \circ \varrho\right)(\kappa)\right|=\left|\mathbf{u}^{\prime}\left(\frac{\mathbf{c}_{2}-\varrho(\kappa)}{\mathbf{c}_{2}-\mathbf{c}_{1}} \mathbf{c}_{1}+\frac{\varrho(\kappa)-\mathbf{c}_{1}}{\mathbf{c}_{2}-\mathbf{c}_{1}} \mathbf{c}_{2}\right)\right| \\
\quad \leq \frac{\mathbf{c}_{2}-\varrho(\kappa)}{\mathbf{c}_{2}-\mathbf{c}_{1}}\left|\mathbf{u}^{\prime}\left(\mathbf{c}_{1}\right)\right|+\frac{\varrho(\kappa)-\mathbf{c}_{1}}{\mathbf{c}_{2}-\mathbf{c}_{1}}\left|\mathbf{u}^{\prime}\left(\mathbf{c}_{2}\right)\right| .
\end{aligned}
$$

Hence, we obtain

$$
\begin{gathered}
\left|\Xi_{1}+\Xi_{2}\right| \leq \frac{\|w\|\left[\mathbf{c}_{1}, \frac{\mathbf{c}_{1}+\mathbf{c}_{2}}{2}\right], \infty}{\left(\mathbf{c}_{2}-\mathbf{c}_{1}\right) \Gamma(v)} \int_{\varrho^{-1}\left(\mathbf{c}_{1}\right)}^{\varrho^{-1}\left(\frac{\mathbf{c}_{1}+\mathbf{c}_{2}}{2}\right)}\left|\int_{\varrho^{-1}\left(\mathbf{c}_{1}\right)}^{\mathcal{K}} \varrho^{\prime}(x)\left(\varrho(x)-\mathbf{c}_{1}\right)^{v-1} d x\right| \\
\quad \times\left[\left(\mathbf{c}_{2}-\varrho(\kappa)\right)\left|\mathbf{u}^{\prime}\left(\mathbf{c}_{1}\right)\right|+\left(\varrho(\kappa)-\mathbf{c}_{1}\right)\left|\mathbf{u}^{\prime}\left(\mathbf{c}_{2}\right)\right|\right] \varrho^{\prime}(\kappa) d \kappa \\
+\frac{\|w\|_{\left[\frac{\mathbf{c}_{1}+\mathbf{c}_{2}}{2}, \mathbf{c}_{2}\right], \infty}}{\left(\mathbf{c}_{2}-\mathbf{c}_{1}\right) \Gamma(v)} \int_{\varrho^{-1}\left(\frac{\mathbf{c}_{1}+\mathbf{c}_{2}}{2}\right)}^{\varrho^{-1}\left(\mathbf{c}_{2}\right)}\left|\int_{\mathcal{K}}^{\varrho^{-1}\left(\mathbf{c}_{2}\right)} \varrho^{\prime}(x)\left(\mathbf{c}_{2}-\varrho(x)\right)^{v-1} d x\right| \\
\quad \times\left[\left(\mathbf{c}_{2}-\varrho(\kappa)\right)\left|\mathbf{u}^{\prime}\left(\mathbf{c}_{1}\right)\right|+\left(\varrho(\kappa)-\mathbf{c}_{1}\right)\left|\mathbf{u}^{\prime}\left(\mathbf{c}_{2}\right)\right|\right] \varrho^{\prime}(\kappa) d \kappa \\
=\frac{\left(\mathbf{c}_{2}-\mathbf{c}_{1}\right)^{v+1}}{2^{v+2} \Gamma(v+3)}\left\{\|w\|_{\left[\mathbf{c}_{1}, \frac{\mathbf{c}_{1}+\mathbf{c}_{2}}{2}\right], \infty}\left[(v+3)\left|\mathbf{u}^{\prime}\left(\mathbf{c}_{1}\right)\right|+(v+1)\left|\mathbf{u}^{\prime}\left(\mathbf{c}_{2}\right)\right|\right]\right. \\
\left.+\|w\|_{\left[\frac{\mathbf{c}_{1}+\mathbf{c}_{2}}{2}, \mathbf{c}_{2}\right], \infty}\left[(v+1)\left|\mathbf{u}^{\prime}\left(\mathbf{c}_{1}\right)\right|+(v+3)\left|\mathbf{u}^{\prime}\left(\mathbf{c}_{2}\right)\right|\right]\right\} \\
\leq \frac{\left(\mathbf{c}_{2}-\mathbf{c}_{1}\right)^{v+1}\|w\|_{\left[\mathbf{c}_{1}, \mathbf{c}_{2}\right], \infty}\left[\left|\mathbf{u}^{\prime}\left(\mathbf{c}_{1}\right)\right|+\left|\mathbf{u}^{\prime}\left(\mathbf{c}_{2}\right)\right|\right],}{2^{v+1} \Gamma(v+2)}
\end{gathered}
$$


where

$$
\begin{aligned}
& \int_{\varrho^{-1}\left(\mathbf{c}_{1}\right)}^{\mathcal{K}} \varrho^{\prime}(x)\left(\varrho(x)-\mathbf{c}_{1}\right)^{v-1} d x=\frac{\left(\varrho(\kappa)-\mathbf{c}_{1}\right)^{v}}{v} ; \\
& \int_{\kappa}^{\varrho^{-1}\left(\mathbf{c}_{2}\right)} \varrho^{\prime}(x)\left(\mathbf{c}_{2}-\varrho(x)\right)^{v-1} d x=\frac{\left(\mathbf{c}_{2}-\varrho(\kappa)\right)^{v}}{v} ; \\
& \int_{\varrho^{-1}\left(\mathbf{c}_{1}\right)}^{\varrho^{-1}\left(\frac{\mathbf{c}_{1}+\mathbf{c}_{2}}{2}\right)}\left(\varrho(\kappa)-\mathbf{c}_{1}\right)^{v+1} \varrho^{\prime}(\kappa) d \kappa=\int_{\varrho^{-1}\left(\frac{\mathbf{c}_{1}+\mathbf{c}_{2}}{2}\right)}^{\varrho^{-1}\left(\mathbf{c}_{2}\right)}\left(\mathbf{c}_{2}-\varrho(\kappa)\right)^{v+1} \varrho^{\prime}(\kappa) d \kappa=\frac{\left(\mathbf{c}_{2}-\mathbf{c}_{1}\right)^{v+2}}{2^{v+2}(v+2)} \\
& \int_{\varrho^{-1}\left(\mathbf{c}_{1}\right)}^{\varrho^{-1}\left(\frac{\mathbf{c}_{1}+\mathbf{c}_{2}}{2}\right)}\left(\varrho(\kappa)-\mathbf{c}_{1}\right)^{v}\left(\mathbf{c}_{2}-\varrho(\kappa)\right) \varrho^{\prime}(\kappa) d \kappa
\end{aligned}
$$

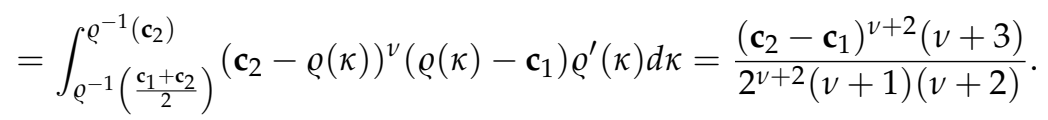

This completes our proof.

Remark 5. From Theorem 2, we can obtain some special cases as follows:

(i) If $\varrho(x)=x$, then inequality (27) becomes

$$
\begin{aligned}
& \mid \mathrm{u}\left(\frac{\boldsymbol{c}_{1}+\boldsymbol{c}_{2}}{2}\right)\left[\begin{array}{c}
{ }^{c_{1}+c_{2}} \\
\left.{ }^{R}\right)+
\end{array}\right. \\
& -\left[w\left(\boldsymbol{c}_{2}\right)\left(\begin{array}{c}
{ }^{\boldsymbol{c}_{1}+\boldsymbol{c}_{2}} \\
\left.{ }^{R}\right)+ \\
\mathcal{I}_{w} \mathrm{u}
\end{array}\right)\left(\boldsymbol{c}_{2}\right)+w\left(\boldsymbol{c}_{1}\right)\left({ }_{w}^{R L} \mathcal{I}_{\left(\frac{c_{1}+c_{2}}{2}\right)-}^{v} \mathrm{u}\right)\left(\boldsymbol{c}_{1}\right)\right] \mid \\
& \leq \frac{\left(c_{2}-c_{1}\right)^{v+1}}{2^{v+2} \Gamma(v+3)}\left\{\|w\|_{\left[c_{1}, \frac{c_{1}+c_{2}}{2}\right], \infty}\left[(v+3)\left|\mathbf{u}^{\prime}\left(c_{1}\right)\right|+(v+1)\left|\mathbf{u}^{\prime}\left(c_{2}\right)\right|\right]\right. \\
& \left.+\|w\|_{\left[\frac{c_{1}+c_{2}}{2}, c_{2}\right], \infty}\left[(v+1)\left|\mathbf{u}^{\prime}\left(c_{1}\right)\right|+(v+3)\left|\mathbf{u}^{\prime}\left(c_{2}\right)\right|\right]\right\} \\
& \leq \frac{\left(c_{2}-c_{1}\right)^{v+1}\|w\|_{\left[c_{1}, c_{2}\right], \infty}}{2^{v+1} \Gamma(v+2)}\left[\left|\mathbf{u}^{\prime}\left(c_{1}\right)\right|+\left|\mathbf{u}^{\prime}\left(\boldsymbol{c}_{2}\right)\right|\right] .
\end{aligned}
$$

(ii) If $\varrho(x)=x$ and $w(x)=1$, then inequality (27) becomes

$$
\begin{aligned}
& \left|\frac{2^{v-1} \Gamma(v+1)}{\left(c_{2}-c_{1}\right)^{v}}\left[\left(\frac{c_{1}+c_{2}}{2}\right)+{ }^{R L} \mathcal{I}^{v} \mathrm{u}\left(\boldsymbol{c}_{2}\right)+{ }^{R L} \mathcal{I}^{v}\left(\frac{c_{1}+c_{2}}{2}\right)-{ }^{v}\left(c_{1}\right)\right]-\mathrm{u}\left(\frac{\boldsymbol{c}_{1}+\boldsymbol{c}_{2}}{2}\right)\right| \\
& \leq \frac{\left(\boldsymbol{c}_{2}-\boldsymbol{c}_{1}\right)^{v+1}}{2^{v+2} \Gamma(v+3)}\left\{\left[(v+3)\left|\mathrm{u}^{\prime}\left(\boldsymbol{c}_{1}\right)\right|+(v+1)\left|\mathrm{u}^{\prime}\left(\boldsymbol{c}_{2}\right)\right|\right]\right. \\
& \left.+\left[(v+1)\left|\mathrm{u}^{\prime}\left(\boldsymbol{c}_{1}\right)\right|+(v+3)\left|\mathrm{u}^{\prime}\left(\boldsymbol{c}_{2}\right)\right|\right]\right\} \leq \frac{\left(\boldsymbol{c}_{2}-\boldsymbol{c}_{1}\right)^{v+1}}{2^{v+1} \Gamma(v+2)}\left[\left|\mathrm{u}^{\prime}\left(\boldsymbol{c}_{1}\right)\right|+\left|\mathrm{u}^{\prime}\left(\boldsymbol{c}_{2}\right)\right|\right],
\end{aligned}
$$

which is already obtained in ([39] [Theorem 5]).

(iii) If $\varrho(x)=x, w(x)=1$ and $v=1$, then inequality (27) becomes

$$
\left|\frac{1}{c_{2}-c_{1}} \int_{c_{1}}^{c_{2}} \mathrm{u}(x) d x-\mathrm{u}\left(\frac{c_{1}+c_{2}}{2}\right)\right| \leq \frac{c_{2}-c_{1}}{8}\left[\left|\mathrm{u}^{\prime}\left(c_{1}\right)\right|+\left|\mathrm{u}^{\prime}\left(c_{2}\right)\right|\right],
$$

which is already obtained in ([45] [Theorem 2.2]). 
Theorem 3. Let $0 \leq \boldsymbol{c}_{1}<\boldsymbol{c}_{2}$, let $\mathrm{u}:\left[\boldsymbol{c}_{1}, \boldsymbol{c}_{2}\right] \subseteq[0, \infty) \rightarrow \mathcal{R}$ be a (continuously) differentiable function on the interval $\left[\boldsymbol{c}_{1}, \boldsymbol{c}_{2}\right]$ such that $\mathrm{u}(x)=\mathrm{u}\left(\boldsymbol{c}_{1}\right)+\int_{\mathcal{c}_{1}}^{x} \mathrm{u}^{\prime}(\kappa) d \kappa$, and let $w:\left[\boldsymbol{c}_{1}, \boldsymbol{c}_{2}\right] \rightarrow \mathcal{R}$ be an integrable, positive and weighted symmetric function with respect to $\frac{c_{1}+c_{2}}{2}$. If, in addition, $\left|\mathrm{u}^{\prime}\right|^{q}$ is convex on $\left[\boldsymbol{c}_{1}, \boldsymbol{c}_{2}\right]$ with $q \geq 1$, and $\varrho$ is an increasing and positive function from $\left[\boldsymbol{c}_{1}, \boldsymbol{c}_{2}\right.$ ) onto itself such that its derivative $Q^{\prime}(x)$ is continuous on $\left(c_{1}, c_{2}\right)$, then for $v>0$, the following inequalities are valid:

$$
\begin{aligned}
&\left|\Xi_{1}+\Xi_{2}\right| \leq \frac{\left(c_{2}-c_{1}\right)^{v+1}}{2^{v+1+\frac{1}{q}}(v+2)^{\frac{1}{q}} \Gamma(v+2)} \\
& \quad \times\left\{\|w\|_{\left[c_{1}, \frac{c_{1}+c_{2}}{2}\right], \infty}\left[(v+3)\left|\mathbf{u}^{\prime}\left(\boldsymbol{c}_{1}\right)\right|^{q}+(v+1)\left|\mathbf{u}^{\prime}\left(\boldsymbol{c}_{2}\right)\right|^{q}\right]^{\frac{1}{q}}\right. \\
&\left.\quad+\|w\|_{\left[\frac{c_{1}+c_{2}}{2}, c_{2}\right], \infty}\left[(v+1)\left|\mathbf{u}^{\prime}\left(\boldsymbol{c}_{1}\right)\right|^{q}+(v+3)\left|\mathbf{u}^{\prime}\left(\boldsymbol{c}_{2}\right)\right|^{q}\right]^{\frac{1}{q}}\right\} \\
& \leq \frac{\left(c_{2}-c_{1}\right)^{v+1}\|w\|_{\left[c_{1}, c_{2}\right], \infty}\left\{\left[(v+3)\left|\mathbf{u}^{\prime}\left(c_{1}\right)\right|^{q}+(v+1)\left|\mathbf{u}^{\prime}\left(c_{2}\right)\right|^{q}\right]^{\frac{1}{q}}\right.}{2^{v+1+\frac{1}{q}}(v+2)^{\frac{1}{q}} \Gamma(v+2)}\left\{+\left[(v+1)\left|\mathbf{u}^{\prime}\left(c_{1}\right)\right|^{q}+(v+3)\left|\mathbf{u}^{\prime}\left(c_{2}\right)\right|^{q}\right]^{\frac{1}{q}}\right\} .
\end{aligned}
$$

Proof. Since $\left|\mathbf{u}^{\prime}\right|^{q}$ is convex on $\left[\mathbf{c}_{1}, \mathbf{c}_{2}\right]$, we get for $\kappa \in\left[\varrho^{-1}\left(\mathbf{c}_{1}\right), \varrho^{-1}\left(\mathbf{c}_{2}\right)\right]$ :

$$
\begin{aligned}
& \left|\left(\mathbf{u}^{\prime} \circ \varrho\right)(\kappa)\right|^{q}=\left|\mathbf{u}^{\prime}\left(\frac{\mathbf{c}_{2}-\varrho(\kappa)}{\mathbf{c}_{2}-\mathbf{c}_{1}} \mathbf{c}_{1}+\frac{\varrho(\kappa)-\mathbf{c}_{1}}{\mathbf{c}_{2}-\mathbf{c}_{1}} \mathbf{c}_{2}\right)\right|^{q} \\
& \quad \leq \frac{\mathbf{c}_{2}-\varrho(\kappa)}{\mathbf{c}_{2}-\mathbf{c}_{1}}\left|\mathbf{u}^{\prime}\left(\mathbf{c}_{1}\right)\right|^{q}+\frac{\varrho(\kappa)-\mathbf{c}_{1}}{\mathbf{c}_{2}-\mathbf{c}_{1}}\left|\mathbf{u}^{\prime}\left(\mathbf{c}_{2}\right)\right|^{q} .
\end{aligned}
$$

By making use of Lemma 2, power mean inequality and convexity of $\left|\mathbf{u}^{\prime}\right|^{q}$, we get

$$
\begin{aligned}
& \left|\Xi_{1}+\Xi_{2}\right| \\
& \leq \frac{1}{\Gamma(v)} \int_{\varrho^{-1}\left(\mathbf{c}_{1}\right)}^{\varrho^{-1}\left(\frac{\mathbf{c}_{1}+\mathbf{c}_{2}}{2}\right)}\left|\int_{\varrho^{-1}\left(\mathbf{c}_{1}\right)}^{\kappa} \varrho^{\prime}(x)\left(\varrho(x)-\mathbf{c}_{1}\right)^{v-1}(w \circ \varrho)(x) d x\right|\left|\left(\mathbf{u}^{\prime} \circ \varrho\right)(\kappa)\right| \varrho^{\prime}(\kappa) d \kappa \\
& +\frac{1}{\Gamma(v)} \int_{\varrho^{-1}\left(\frac{\mathbf{c}_{1}+\mathbf{c}_{2}}{2}\right)}^{\varrho^{-1}\left(\mathbf{c}_{2}\right)}\left|\int_{\mathcal{K}}^{\varrho^{-1}\left(\mathbf{c}_{2}\right)} \varrho^{\prime}(x)\left(\mathbf{c}_{2}-\varrho(x)\right)^{v-1}(w \circ \varrho)(x) d x\right|\left|\left(\mathbf{u}^{\prime} \circ \varrho\right)(\kappa)\right| \varrho^{\prime}(\kappa) d \kappa \\
& \leq \frac{1}{\Gamma(v)}\left(\int_{\varrho^{-1}\left(\mathbf{c}_{1}\right)}^{\varrho^{-1}\left(\frac{\mathbf{c}_{1}+\mathbf{c}_{2}}{2}\right)}\left|\int_{\varrho^{-1}\left(\mathbf{c}_{1}\right)}^{\kappa} \varrho^{\prime}(x)\left(\varrho(x)-\mathbf{c}_{1}\right)^{\nu-1}(w \circ \varrho)(x) d x\right| \varrho^{\prime}(\kappa) d \kappa\right)^{1-\frac{1}{q}} \\
& \times\left(\int_{\varrho^{-1}\left(\mathbf{c}_{1}\right)}^{\varrho^{-1}\left(\frac{\mathbf{c}_{1}+\mathbf{c}_{2}}{2}\right)}\left|\int_{\varrho^{-1}\left(\mathbf{c}_{1}\right)}^{\kappa} \varrho^{\prime}(x)\left(\varrho(x)-\mathbf{c}_{1}\right)^{v-1}(w \circ \varrho)(x) d x\right|\left|\left(\mathbf{u}^{\prime} \circ \varrho\right)(\kappa)\right|^{q} \varrho^{\prime}(\kappa) d \kappa\right)^{\frac{1}{q}} \\
& +\frac{1}{\Gamma(v)}\left(\int_{\varrho^{-1}\left(\frac{\mathbf{c}_{1}+\mathbf{c}_{2}}{2}\right)}^{\varrho^{-1}\left(\mathbf{c}_{2}\right)}\left|\int_{\mathcal{K}}^{\varrho^{-1}\left(\mathbf{c}_{2}\right)} \varrho^{\prime}(x)\left(\mathbf{c}_{2}-\varrho(x)\right)^{\nu-1}(w \circ \varrho)(x) d x\right| \varrho^{\prime}(\kappa) d \kappa\right)^{1-\frac{1}{q}} \\
& \times\left(\int_{\varrho^{-1}\left(\frac{\mathfrak{c}_{1}+\mathbf{c}_{2}}{2}\right)}\left|\int_{\kappa}^{\varrho^{-1}\left(\mathbf{c}_{2}\right)} \varrho^{\prime}(x)\left(\mathbf{c}_{2}-\varrho(x)\right)^{\nu-1}(w \circ \varrho)(x) d x\right|\left|\left(\mathbf{u}^{\prime} \circ \varrho\right)(\kappa)\right|^{q} \varrho^{\prime}(\kappa) d \kappa\right)^{\frac{1}{q}}
\end{aligned}
$$




$$
\begin{aligned}
& \leq \frac{\|w\|\left[\mathrm{c}_{1}, \frac{\mathrm{c}_{1}+\mathrm{c}_{2}}{2}\right], \infty}{\Gamma(v)} \\
& \times\left(\int_{\varrho^{-1}\left(\mathbf{c}_{1}\right)}^{\varrho^{-1}\left(\frac{\mathbf{c}_{1}+\mathbf{c}_{2}}{2}\right)}\left|\int_{\varrho^{-1}\left(\mathbf{c}_{1}\right)}^{\kappa} \varrho^{\prime}(x)\left(\varrho(x)-\mathbf{c}_{1}\right)^{\nu-1} d x\right| \varrho^{\prime}(\kappa) d \kappa\right)^{1-\frac{1}{q}} \\
& \times\left(\int_{\varrho^{-1}\left(\mathbf{c}_{1}\right)}^{\varrho^{-1}\left(\frac{\mathbf{c}_{1}+\mathbf{c}_{2}}{2}\right)}\left|\int_{\varrho^{-1}\left(\mathbf{c}_{1}\right)}^{\kappa} \varrho^{\prime}(x)\left(\varrho(x)-\mathbf{c}_{1}\right)^{\nu-1} d x\right|\left|\left(\mathbf{u}^{\prime} \circ \varrho\right)(\kappa)\right|^{q} \varrho^{\prime}(\kappa) d \kappa\right)^{\frac{1}{q}} \\
& +\frac{\|w\|_{\left[\frac{\mathbf{c}_{1}+\mathbf{c}_{2}}{2}, \mathbf{c}_{2}\right], \infty}}{\Gamma(v)} \\
& \times\left(\int_{\varrho^{-1}\left(\frac{\mathbf{c}_{1}+\mathbf{c}_{2}}{2}\right)}^{\varrho^{-1}\left(\mathbf{c}_{2}\right)}\left|\int_{\mathcal{K}}^{\varrho^{-1}\left(\mathbf{c}_{2}\right)} \varrho^{\prime}(x)\left(\mathbf{c}_{2}-\varrho(x)\right)^{v-1} d x\right| \varrho^{\prime}(\kappa) d \kappa\right)^{1-\frac{1}{q}} \\
& \times\left(\int_{\varrho^{-1}\left(\frac{\boldsymbol{c}_{1}+\mathbf{c}_{2}}{2}\right)}^{\varrho^{-1}\left(\mathbf{c}_{2}\right)}\left|\int_{\kappa}^{\varrho^{-1}\left(\mathbf{c}_{2}\right)} \varrho^{\prime}(x)\left(\mathbf{c}_{2}-\varrho(x)\right)^{v-1} d x\right|\left|\left(\mathbf{u}^{\prime} \circ \varrho\right)(\kappa)\right|^{q} \varrho^{\prime}(\kappa) d \kappa\right)^{\frac{1}{q}} \\
& \leq \frac{\|w\|_{\left[\mathbf{c}_{1}, \frac{\mathbf{c}_{1}+\mathbf{c}_{2}}{2}\right], \infty}}{\Gamma(v)} \\
& \left(\int_{\varrho^{-1}\left(\mathbf{c}_{1}\right)}^{\varrho^{-1}\left(\frac{\mathbf{c}_{1}+\mathbf{c}_{2}}{2}\right)}\left|\int_{\varrho^{-1}\left(\mathbf{c}_{1}\right)}^{\kappa} \varrho^{\prime}(x)\left(\varrho(x)-\mathbf{c}_{1}\right)^{v-1} d x\right| \varrho^{\prime}(\kappa) d \kappa\right)^{1-\frac{1}{q}} \\
& \times\left[\int_{\varrho^{-1}\left(\mathbf{c}_{1}\right)}^{\varrho^{-1}\left(\frac{\mathbf{c}_{1}+\mathbf{c}_{2}}{2}\right)}\left|\int_{\varrho^{-1}\left(\mathbf{c}_{1}\right)}^{\mathcal{K}} \varrho^{\prime}(x)\left(\varrho(x)-\mathbf{c}_{1}\right)^{v-1} d x\right|\right. \\
& \left.\times\left(\frac{\mathbf{c}_{2}-\varrho(\kappa)}{\mathbf{c}_{2}-\mathbf{c}_{1}}\left|\mathbf{u}^{\prime}\left(\mathbf{c}_{1}\right)\right|^{q}+\frac{\varrho(\kappa)-\mathbf{c}_{1}}{\mathbf{c}_{2}-\mathbf{c}_{1}}\left|\mathbf{u}^{\prime}\left(\mathbf{c}_{2}\right)\right|^{q}\right) \varrho^{\prime}(\kappa) d \kappa\right]^{\frac{1}{q}} \\
& +\frac{\|w\|\left[\frac{\mathbf{c}_{1}+\mathbf{c}_{2}}{2}, \mathbf{c}_{2}\right], \infty}{\Gamma(v)}\left(\int_{\varrho^{-1}\left(\frac{\mathbf{c}_{1}+\mathbf{c}_{2}}{2}\right)}^{\varrho^{-1}\left(\mathbf{c}_{2}\right)}\left|\int_{\kappa}^{\varrho^{-1}\left(\mathbf{c}_{2}\right)} \varrho^{\prime}(x)\left(\mathbf{c}_{2}-\varrho(x)\right)^{\nu-1} d x\right| \varrho^{\prime}(\kappa) d \kappa\right)^{1-\frac{1}{q}} \\
& \times\left[\int_{\varrho^{-1}\left(\frac{\mathbf{c}_{1}+\mathbf{c}_{2}}{2}\right)}^{\varrho^{-1}\left(\mathbf{c}_{2}\right)}\left|\int_{\mathcal{K}}^{\varrho^{-1}\left(\mathbf{c}_{2}\right)} \varrho^{\prime}(x)\left(\mathbf{c}_{2}-\varrho(x)\right)^{\nu-1} d x\right|\right. \\
& \left.\times\left(\frac{\mathbf{c}_{2}-\varrho(\kappa)}{\mathbf{c}_{2}-\mathbf{c}_{1}}\left|\mathbf{u}^{\prime}\left(\mathbf{c}_{1}\right)\right|^{q}+\frac{\varrho(\kappa)-\mathbf{c}_{1}}{\mathbf{c}_{2}-\mathbf{c}_{1}}\left|\mathbf{u}^{\prime}\left(\mathbf{c}_{2}\right)\right|^{q}\right) \varrho^{\prime}(\kappa) d \kappa\right]^{\frac{1}{q}} \\
& =\frac{\left(\mathbf{c}_{2}-\mathbf{c}_{1}\right)^{v+1}}{2^{v+1+\frac{1}{q}}(v+2)^{\frac{1}{q}} \Gamma(v+2)} \\
& \times\left\{\|w\|_{\left[\mathbf{c}_{1}, \frac{\mathbf{c}_{1}+\mathbf{c}_{2}}{2}\right], \infty}\left[(v+3)\left|\mathbf{u}^{\prime}\left(\mathbf{c}_{1}\right)\right|^{q}+(v+1)\left|\mathbf{u}^{\prime}\left(\mathbf{c}_{2}\right)\right|^{q}\right]^{\frac{1}{q}}\right. \\
& \left.+\|w\|_{\left[\frac{\mathbf{c}_{1}+\mathbf{c}_{2}}{2}, \mathbf{c}_{2}\right], \infty}\left[(v+1)\left|\mathbf{u}^{\prime}\left(\mathbf{c}_{1}\right)\right|^{q}+(v+3)\left|\mathbf{u}^{\prime}\left(\mathbf{c}_{2}\right)\right|^{q}\right]^{\frac{1}{q}}\right\}
\end{aligned}
$$




$$
\begin{aligned}
\leq \frac{\left(\mathbf{c}_{2}-\mathbf{c}_{1}\right)^{v+1}\|w\|_{\left[\mathbf{c}_{1}, \mathbf{c}_{2}\right], \infty}}{2^{v+1+\frac{1}{q}}(v+2)^{\frac{1}{q}} \Gamma(v+2)}\left\{\left[(v+3)\left|\mathbf{u}^{\prime}\left(\mathbf{c}_{1}\right)\right|^{q}+(v+1)\left|\mathbf{u}^{\prime}\left(\mathbf{c}_{2}\right)\right|^{q}\right]^{\frac{1}{q}}\right. \\
\left.+\left[(v+1)\left|\mathbf{u}^{\prime}\left(\mathbf{c}_{1}\right)\right|^{q}+(v+3)\left|\mathbf{u}^{\prime}\left(\mathbf{c}_{2}\right)\right|^{q}\right]^{\frac{1}{q}}\right\},
\end{aligned}
$$

where it is easily seen that

$$
\begin{aligned}
\int_{\varrho^{-1}\left(\mathbf{c}_{1}\right)}^{\varrho^{-1}\left(\frac{\mathbf{c}_{1}+\mathbf{c}_{2}}{2}\right)} \mid & \int_{\varrho^{-1}\left(\mathbf{c}_{1}\right)}^{\kappa} \varrho^{\prime}(x)\left(\varrho(x)-\mathbf{c}_{1}\right)^{v-1} d x \mid \varrho^{\prime}(\kappa) d \kappa \\
& =\int_{\varrho^{-1}\left(\frac{\mathbf{c}_{1}+\mathbf{c}_{2}}{2}\right)}^{\varrho^{-1}\left(\mathbf{c}_{2}\right)}\left|\int_{\kappa}^{\varrho^{-1}\left(\mathbf{c}_{2}\right)} \varrho^{\prime}(x)\left(\mathbf{c}_{2}-\varrho(x)\right)^{v-1} d x\right| \varrho^{\prime}(\kappa) d \kappa=\frac{\left(\mathbf{c}_{2}-\mathbf{c}_{1}\right)^{v+1}}{2^{v+1} v(v+1)} .
\end{aligned}
$$

Hence, the proof is completed.

Remark 6. From Theorem 3, we can obtain some special cases as follows:

(i) If $\varrho(x)=x$, then inequality (34) becomes

$$
\begin{aligned}
& \mid \mathrm{u}\left(\frac{\boldsymbol{c}_{1}+\boldsymbol{c}_{2}}{2}\right)\left[\left(\frac{\boldsymbol{c}_{1}+c_{2}}{2}\right)+{ }^{R L} \mathcal{I}^{v} w\left(\boldsymbol{c}_{2}\right)+{ }^{R L} \mathcal{I}_{\left(\frac{c_{1}+c_{2}}{2}\right)-}^{v} w\left(\boldsymbol{c}_{1}\right)\right]
\end{aligned}
$$

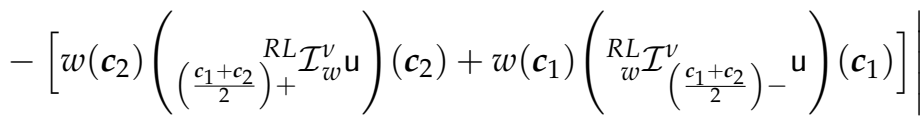

$$
\begin{aligned}
& \leq \frac{\left(c_{2}-c_{1}\right)^{v+1}}{2^{v+1+\frac{1}{q}}(v+2)^{\frac{1}{q}} \Gamma(v+2)} \\
& \times\left\{\|w\|_{\left[c_{1}, \frac{c_{1}+c_{2}}{2}\right], \infty}\left[(v+3)\left|\mathbf{u}^{\prime}\left(\boldsymbol{c}_{1}\right)\right|^{q}+(v+1)\left|\mathbf{u}^{\prime}\left(\boldsymbol{c}_{2}\right)\right|^{q}\right]^{\frac{1}{q}}\right. \\
& \left.+\|w\|_{\left[\frac{c_{1}+c_{2}}{2}, c_{2}\right], \infty}\left[(v+1)\left|\mathbf{u}^{\prime}\left(c_{1}\right)\right|^{q}+(v+3)\left|\mathbf{u}^{\prime}\left(\boldsymbol{c}_{2}\right)\right|^{q}\right]^{\frac{1}{q}}\right\} \\
& \leq \frac{\left(c_{2}-c_{1}\right)^{v+1}\|w\|_{\left[c_{1}, c_{2}\right], \infty}}{2^{v+1+\frac{1}{q}}(v+2)^{\frac{1}{q}} \Gamma(v+2)}\left\{\left[(v+3)\left|\mathbf{u}^{\prime}\left(\boldsymbol{c}_{1}\right)\right|^{q}+(v+1)\left|\mathbf{u}^{\prime}\left(\boldsymbol{c}_{2}\right)\right|^{q}\right]^{\frac{1}{q}}\right. \\
& \left.+\left[(v+1)\left|\mathbf{u}^{\prime}\left(\boldsymbol{c}_{1}\right)\right|^{q}+(v+3)\left|\mathbf{u}^{\prime}\left(\boldsymbol{c}_{2}\right)\right|^{q}\right]^{\frac{1}{q}}\right\} .
\end{aligned}
$$

(ii) If $\varrho(x)=x$ and $w(x)=1$, then inequality (34) becomes

$$
\begin{aligned}
& \left|\frac{2^{v-1} \Gamma(v+1)}{\left(\boldsymbol{c}_{2}-\boldsymbol{c}_{1}\right)^{v}}\left[\left(\frac{\boldsymbol{c}_{1}+c_{2}}{2}\right)+{ }^{R L} \mathcal{I}^{v} \mathrm{u}\left(\boldsymbol{c}_{2}\right)+{ }^{R L} \mathcal{I}_{\left(\frac{c_{1}+c_{2}}{2}\right)-}^{v} \mathrm{u}\left(\boldsymbol{c}_{1}\right)\right]-\mathrm{u}\left(\frac{\boldsymbol{c}_{1}+\boldsymbol{c}_{2}}{2}\right)\right| \\
& \leq \frac{\left(\boldsymbol{c}_{2}-\boldsymbol{c}_{1}\right)^{v+1}}{2^{v+1+\frac{1}{q}}(v+2)^{\frac{1}{q}} \Gamma(v+2)}\left\{\left[(v+3)\left|\mathbf{u}^{\prime}\left(\boldsymbol{c}_{1}\right)\right|^{q}+(v+1)\left|\mathbf{u}^{\prime}\left(\boldsymbol{c}_{2}\right)\right|^{q}\right]^{\frac{1}{q}}\right. \\
& \left.+\left[(v+1)\left|\mathbf{u}^{\prime}\left(\boldsymbol{c}_{1}\right)\right|^{q}+(v+3)\left|\mathbf{u}^{\prime}\left(\boldsymbol{c}_{2}\right)\right|^{q}\right]^{\frac{1}{q}}\right\},
\end{aligned}
$$

which is already obtained in ([39] [Theorem 5]). 
(iii) If $\varrho(x)=x, w(x)=1$ and $v=1$, then inequality (34) becomes

$$
\begin{aligned}
& \left|\frac{1}{c_{2}-c_{1}} \int_{c_{1}}^{c_{2}} \mathrm{u}(x) d x-\mathrm{u}\left(\frac{c_{1}+c_{2}}{2}\right)\right| \\
& \quad \leq \frac{c_{2}-c_{1}}{8 \sqrt[q]{3}}\left\{\left[\left|\mathrm{u}^{\prime}\left(c_{1}\right)\right|^{q}+2\left|\mathrm{u}^{\prime}\left(c_{2}\right)\right|^{q}\right]^{\frac{1}{q}}+\left[2\left|\mathrm{u}^{\prime}\left(c_{1}\right)\right|^{q}+\left|\mathrm{u}^{\prime}\left(\boldsymbol{c}_{2}\right)\right|^{q}\right]^{\frac{1}{q}}\right\} .
\end{aligned}
$$

Theorem 4. Let $0 \leq \boldsymbol{c}_{1}<c_{2}$, let $\mathrm{u}:\left[\boldsymbol{c}_{1}, \boldsymbol{c}_{2}\right] \subseteq[0, \infty) \rightarrow \mathcal{R}$ be a (continuously) differentiable function on the interval $\left[\boldsymbol{c}_{1}, \boldsymbol{c}_{2}\right]$ such that $\mathrm{u}(x)=\mathrm{u}\left(\boldsymbol{c}_{1}\right)+\int_{\mathcal{c}_{1}}^{x} \mathrm{u}^{\prime}(\kappa) d \kappa$, and let $w:\left[\boldsymbol{c}_{1}, \boldsymbol{c}_{2}\right] \rightarrow \mathcal{R}$ be an integrable, positive and weighted symmetric function with respect to $\frac{c_{1}+c_{2}}{2}$. If, in addition, $\left|\mathbf{u}^{\prime}\right|^{q}$ is convex on $\left[\boldsymbol{c}_{1}, \boldsymbol{c}_{2}\right]$ with $\frac{1}{p}+\frac{1}{q}=1$ and $q>1$, and $\varrho$ is an increasing and positive function from $\left[\boldsymbol{c}_{1}, \boldsymbol{c}_{2}\right)$ onto itself such that its derivative $\varrho^{\prime}(x)$ is continuous on $\left(\boldsymbol{c}_{1}, \boldsymbol{c}_{2}\right)$, then for $v>0$ the following inequalities are valid:

$$
\begin{gathered}
\left|\Xi_{1}+\Xi_{2}\right| \leq \frac{\left(c_{2}-c_{1}\right)^{v+1}}{2^{v+1+\frac{2}{q}}(p v+1)^{\frac{1}{p}} \Gamma(v+1)}\left\{\|w\|_{\left[c_{1}, \frac{c_{1}+c_{2}}{2}\right], \infty}\right. \\
\left.\times\left[3\left|\mathbf{u}^{\prime}\left(c_{1}\right)\right|^{q}+\left|\mathbf{u}^{\prime}\left(c_{2}\right)\right|^{q}\right]^{\frac{1}{q}}+\|w\| \|_{\left[\frac{c_{1}+c_{2}}{2}, c_{2}\right], \infty}\left[\left|\mathbf{u}^{\prime}\left(c_{1}\right)\right|^{q}+3\left|\mathbf{u}^{\prime}\left(c_{2}\right)\right|^{q}\right]^{\frac{1}{q}}\right\} \\
\leq \frac{\left(c_{2}-c_{1}\right)^{v+1}\|w\|}{2^{v+1+\frac{2}{q}}(p v+1)^{\frac{1}{p}} \Gamma(v+1)} \\
\times\left\{\left[3\left|\mathbf{u}^{\prime}\left(c_{1}\right)\right|^{q}+\left|\mathbf{u}^{\prime}\left(c_{2}\right)\right|^{q}\right]^{\frac{1}{q}}+\left[\left|\mathbf{u}^{\prime}\left(c_{1}\right)\right|^{q}+3\left|\mathbf{u}^{\prime}\left(c_{2}\right)\right|^{q}\right]^{\frac{1}{q}}\right\} .
\end{gathered}
$$

Proof. Since $\left|\mathbf{u}^{\prime}\right|^{q}$ is convex on $\left[\mathbf{c}_{1}, \mathbf{c}_{2}\right]$, we get for $\kappa \in\left[\varrho^{-1}\left(\mathbf{c}_{1}\right), \varrho^{-1}\left(\mathbf{c}_{2}\right)\right]$ :

$$
\begin{aligned}
\left|\left(\mathbf{u}^{\prime} \circ \varrho\right)(\kappa)\right|^{q}=\left|\mathbf{u}^{\prime}\left(\frac{\mathbf{c}_{2}-\varrho(\kappa)}{\mathbf{c}_{2}-\mathbf{c}_{1}} \mathbf{c}_{1}+\frac{\varrho(\kappa)-\mathbf{c}_{1}}{\mathbf{c}_{2}-\mathbf{c}_{1}} \mathbf{c}_{2}\right)\right|^{q} \\
\quad \leq \frac{\mathbf{c}_{2}-\varrho(\kappa)}{\mathbf{c}_{2}-\mathbf{c}_{1}}\left|\mathbf{u}^{\prime}\left(\mathbf{c}_{1}\right)\right|^{q}+\frac{\varrho(\kappa)-\mathbf{c}_{1}}{\mathbf{c}_{2}-\mathbf{c}_{1}}\left|\mathbf{u}^{\prime}\left(\mathbf{c}_{2}\right)\right|^{q} .
\end{aligned}
$$

By using Lemma 2, Hölder's inequality, convexity of $\left|\mathbf{u}^{\prime}\right|^{q}$ and properties of modulus, we have

$$
\begin{aligned}
&\left|\Xi_{1}+\Xi_{2}\right| \leq \frac{1}{\Gamma(v)} \int_{\varrho^{-1}\left(\mathbf{c}_{1}\right)}^{\varrho^{-1}\left(\frac{\mathbf{c}_{1}+\mathbf{c}_{2}}{2}\right) \mid}\left|\int_{\varrho^{-1}\left(\mathbf{c}_{1}\right)}^{\kappa} \varrho^{\prime}(x)\left(\varrho(x)-\mathbf{c}_{1}\right)^{v-1}(w \circ \varrho)(x) d x\right| \\
& \times\left|\left(\mathbf{u}^{\prime} \circ \varrho\right)(\kappa)\right| \varrho^{\prime}(\kappa) d \kappa \\
&+\frac{1}{\Gamma(v)} \int_{\varrho^{-1}\left(\frac{\mathbf{c}_{1}+\mathbf{c}_{2}}{2}\right) \mid}^{\varrho^{-1}\left(\mathbf{c}_{2}\right)}\left|\int_{\mathcal{\kappa}}^{\varrho^{-1}\left(\mathbf{c}_{2}\right)} \varrho^{\prime}(x)\left(\mathbf{c}_{2}-\varrho(x)\right)^{v-1}(w \circ \varrho)(x) d x\right|\left|\left(\mathbf{u}^{\prime} \circ \varrho\right)(\kappa)\right| \varrho^{\prime}(\kappa) d \kappa \\
& \leq \frac{1}{\Gamma(v)}\left(\int_{\varrho^{-1}\left(\mathbf{c}_{1}\right)}^{\varrho^{-1}\left(\frac{\mathbf{c}_{1}+\mathbf{c}_{2}}{2}\right) \mid}\left|\int_{\varrho^{-1}\left(\mathbf{c}_{1}\right)}^{\kappa} \varrho^{\prime}(x)\left(\varrho(x)-\mathbf{c}_{1}\right)^{v-1}(w \circ \varrho)(x) d x\right|^{p} \varrho^{\prime}(\kappa) d \kappa\right)^{\frac{1}{p}}
\end{aligned}
$$




$$
\begin{aligned}
& \times\left(\int_{\varrho^{-1}\left(\mathbf{c}_{1}\right)}^{\varrho^{-1}\left(\frac{\mathbf{c}_{1}+\mathbf{c}_{2}}{2}\right)}\left|\left(\mathbf{u}^{\prime} \circ \varrho\right)(\kappa)\right|^{q} \varrho^{\prime}(\kappa) d \kappa\right)^{\frac{1}{q}} \\
& +\frac{1}{\Gamma(v)}\left(\int_{\varrho^{-1}\left(\frac{\varrho^{-1}+\mathbf{c}_{2}}{2}\right)}\left|\int_{\kappa}^{\varrho^{-1}\left(\mathbf{c}_{2}\right)} \varrho^{\prime}(x)\left(\mathbf{c}_{2}-\varrho(x)\right)^{v-1}(w \circ \varrho)(x) d x\right|^{p} \varrho^{\prime}(\kappa) d \kappa\right)^{\frac{1}{p}} \\
& \times\left(\int_{\varrho^{-1}\left(\frac{\mathbf{c}_{1}+\mathbf{c}_{2}}{2}\right)}^{\varrho^{-1}\left(\mathbf{c}_{2}\right)}\left|\left(\mathbf{u}^{\prime} \circ \varrho\right)(\kappa)\right|^{q} \varrho^{\prime}(\kappa) d \kappa\right)^{\frac{1}{q}} \\
& \leq \frac{\|w\|_{\left[\mathbf{c}_{1}, \frac{\mathbf{c}_{1}+\mathbf{c}_{2}}{2}\right], \infty}}{\Gamma(v)} \\
& \times\left(\int_{\varrho^{-1}\left(\mathbf{c}_{1}\right)}^{\varrho^{-1}\left(\frac{\mathbf{c}_{1}+\mathbf{c}_{2}}{2}\right)}\left|\int_{\varrho^{-1}\left(\mathbf{c}_{1}\right)}^{\mathcal{K}} \varrho^{\prime}(x)\left(\varrho(x)-\mathbf{c}_{1}\right)^{v-1} d x\right|^{p} \varrho^{\prime}(\kappa) d \kappa\right)^{\frac{1}{p}} \\
& \times\left(\int_{\varrho^{-1}\left(\mathbf{c}_{1}\right)}^{\varrho^{-1}\left(\frac{\mathbf{c}_{1}+\mathbf{c}_{2}}{2}\right)}\left|\left(\mathbf{u}^{\prime} \circ \varrho\right)(\kappa)\right|^{q} \varrho^{\prime}(\kappa) d \kappa\right)^{\frac{1}{q}}+\frac{\|w\|\left[\frac{\mathbf{c}_{1}+\mathbf{c}_{2}}{2}, \mathbf{c}_{2}\right], \infty}{\Gamma(v)} \\
& \times\left(\int_{\varrho^{-1}\left(\frac{\varrho^{-1}+\mathbf{c}_{2}}{2}\right)}\left|\int_{\mathcal{\kappa}}^{\varrho^{-1}\left(\mathbf{c}_{2}\right)} \varrho^{\prime}(x)\left(\mathbf{c}_{2}-\varrho(x)\right)^{\nu-1} d x\right|^{p} \varrho^{\prime}(\kappa) d \kappa\right)^{\frac{1}{p}} \\
& \times\left(\int_{\varrho^{-1}\left(\frac{\mathbf{c}_{1}+\mathbf{c}_{2}}{2}\right)}^{\varrho^{-1}\left(\mathbf{c}_{2}\right.}\left|\left(\mathbf{u}^{\prime} \circ \varrho\right)(\kappa)\right|^{q} \varrho^{\prime}(\kappa) d \kappa\right)^{\frac{1}{q}} \\
& \leq \frac{\|w\|\left[\mathbf{c}_{1}, \frac{\mathbf{c}_{1}+\mathbf{c}_{2}}{2}\right], \infty}{\Gamma(v)} \\
& \times\left(\int_{\varrho^{-1}\left(\mathbf{c}_{1}\right)}^{\varrho^{-1}\left(\frac{\mathbf{c}_{1}+\mathbf{c}_{2}}{2}\right)}\left|\int_{\varrho^{-1}\left(\mathbf{c}_{1}\right)}^{\kappa} \varrho^{\prime}(x)\left(\varrho(x)-\mathbf{c}_{1}\right)^{v-1} d x\right|^{p} \varrho^{\prime}(\kappa) d \kappa\right)^{\frac{1}{p}} \\
& \times\left[\int_{\varrho^{-1}\left(\mathbf{c}_{1}\right)}^{\varrho^{-1}\left(\frac{\mathbf{c}_{1}+\mathbf{c}_{2}}{2}\right)}\left(\frac{\mathbf{c}_{2}-\varrho(\kappa)}{\mathbf{c}_{2}-\mathbf{c}_{1}}\left|\mathbf{u}^{\prime}\left(\mathbf{c}_{1}\right)\right|^{q}+\frac{\varrho(\kappa)-\mathbf{c}_{1}}{\mathbf{c}_{2}-\mathbf{c}_{1}}\left|\mathbf{u}^{\prime}\left(\mathbf{c}_{2}\right)\right|^{q}\right) \varrho^{\prime}(\kappa) d \kappa\right]^{\frac{1}{q}} \\
& +\frac{\|w\|_{\left[\frac{\mathbf{c}_{1}+\mathbf{c}_{2}}{2}, \mathbf{c}_{2}\right], \infty}}{\Gamma(v)} \\
& \times\left(\int_{\varrho^{-1}\left(\frac{\mathbf{c}_{1}+\mathbf{c}_{2}}{2}\right)}^{\varrho^{-1}\left(\mathbf{c}_{2}\right)}\left|\int_{\kappa}^{\varrho^{-1}\left(\mathbf{c}_{2}\right)} \varrho^{\prime}(x)\left(\mathbf{c}_{2}-\varrho(x)\right)^{\nu-1} d x\right|^{p} \varrho^{\prime}(\kappa) d \kappa\right)^{\frac{1}{p}} \\
& \times\left[\int_{\varrho^{-1}\left(\frac{\varrho_{1}+\mathbf{c}_{2}}{2}\right)}^{\varrho^{-1}\left(\mathbf{c}_{2}\right)}\left(\frac{\mathbf{c}_{2}-\varrho(\kappa)}{\mathbf{c}_{2}-\mathbf{c}_{1}}\left|\mathbf{u}^{\prime}\left(\mathbf{c}_{1}\right)\right|^{q}+\frac{\varrho(\kappa)-\mathbf{c}_{1}}{\mathbf{c}_{2}-\mathbf{c}_{1}}\left|\mathbf{u}^{\prime}\left(\mathbf{c}_{2}\right)\right|^{q}\right) \varrho^{\prime}(\kappa) d \kappa\right]^{\frac{1}{q}}
\end{aligned}
$$




$$
\begin{aligned}
&=\frac{\left(\mathbf{c}_{2}-\mathbf{c}_{1}\right)^{v+1}}{2^{v+1+\frac{2}{q}}(p v+1)^{\frac{1}{p}} \Gamma(v+1)}\left\{\|w\|_{\left[\mathbf{c}_{1}, \frac{\mathbf{c}_{1}+\mathbf{c}_{2}}{2}\right], \infty}\left[3\left|\mathbf{u}^{\prime}\left(\mathbf{c}_{1}\right)\right|^{q}+\left|\mathbf{u}^{\prime}\left(\mathbf{c}_{2}\right)\right|^{q}\right]^{\frac{1}{q}}\right. \\
&+\|w\|_{\left[\frac{\mathbf{c}_{1}+\mathbf{c}_{2}}{2}, \mathbf{c}_{2}\right], \infty}\left[\left|\mathbf{u}^{\prime}\left(\mathbf{c}_{1}\right)\right|^{q}+3\left|\mathbf{u}^{\prime}\left(\mathbf{c}_{2}\right)\right|^{q}\right]^{\frac{1}{q}} \\
& \leq \frac{\left(\mathbf{c}_{2}-\mathbf{c}_{1}\right)^{v+1}\|w\|}{2^{v+1+\frac{2}{q}}(p v+1)^{\frac{1}{p}} \Gamma(v+1)} \\
& \times\left\{\left[3\left|\mathbf{u}^{\prime}\left(\mathbf{c}_{1}\right)\right|^{q}+\left|\mathbf{u}^{\prime}\left(\mathbf{c}_{2}\right)\right|^{q}\right]^{\frac{1}{q}}+\left[\left|\mathbf{u}^{\prime}\left(\mathbf{c}_{1}\right)\right|^{q}+3\left|\mathbf{u}^{\prime}\left(\mathbf{c}_{2}\right)\right|^{q}\right]^{\frac{1}{q}}\right\},
\end{aligned}
$$

where we used the identity

$$
\begin{aligned}
\int_{\varrho^{-1}\left(\mathbf{c}_{1}\right)}^{\varrho^{-1}\left(\frac{\mathbf{c}_{1}+\mathbf{c}_{2}}{2}\right)} \mid & \left|\int_{\varrho^{-1}\left(\mathbf{c}_{1}\right)}^{\kappa} \varrho^{\prime}(x)\left(\varrho(x)-\mathbf{c}_{1}\right)^{v-1} d x\right|^{p} \varrho^{\prime}(\kappa) d \kappa \\
= & \int_{\varrho^{-1}\left(\frac{\mathfrak{c}_{1}+\mathbf{c}_{2}}{2}\right)}^{\varrho^{-1}\left(\mathbf{c}_{2}\right)}\left|\int_{\kappa}^{\varrho^{-1}\left(\mathbf{c}_{2}\right)} \varrho^{\prime}(x)\left(\mathbf{c}_{2}-\varrho(x)\right)^{v-1} d x\right|^{p} \varrho^{\prime}(\kappa) d \kappa=\frac{\left(\mathbf{c}_{2}-\mathbf{c}_{1}\right)^{p v+1}}{2^{p v+1}(p v+1) v^{p}} .
\end{aligned}
$$

This ends our proof.

Remark 7. From Theorem 4, we can obtain some special cases as follows:

(i) If $\varrho(x)=x$, then inequality (40) becomes

$$
\begin{aligned}
& \mid \mathrm{u}\left(\frac{\boldsymbol{c}_{1}+\boldsymbol{c}_{2}}{2}\right)\left[\left(\frac{\boldsymbol{c}_{1}+c_{2}}{2}\right)+{ }^{R L} \mathcal{I}^{v} w\left(\boldsymbol{c}_{2}\right)+{ }^{R L} \mathcal{I}_{\left(\frac{c_{1}+c_{2}}{2}\right)-}^{v} w\left(\boldsymbol{c}_{1}\right)\right] \\
& -\left[w\left(\boldsymbol{c}_{2}\right)\left({ }_{\left(\frac{c_{1}+c_{2}}{2}\right)+}^{R L} \mathcal{I}_{w}^{v} \mathbf{u}\right)\left(\boldsymbol{c}_{2}\right)+w\left(\boldsymbol{c}_{1}\right)\left({ }_{w}^{R L} \mathcal{I}_{\left(\frac{c_{1}+c_{2}}{2}\right)-}^{v} \mathbf{u}\right)\left(\boldsymbol{c}_{1}\right)\right] \\
& \leq \frac{\left(\boldsymbol{c}_{2}-\boldsymbol{c}_{1}\right)^{v+1}}{2^{v+1+\frac{2}{\bar{q}}}(p v+1)^{\frac{1}{p}} \Gamma(v+1)}\left\{\|w\|_{\left[c_{1}, \frac{c_{1}+c_{2}}{2}\right], \infty}\left[3\left|\mathbf{u}^{\prime}\left(\boldsymbol{c}_{1}\right)\right|^{q}+\left|\mathbf{u}^{\prime}\left(\boldsymbol{c}_{2}\right)\right|^{q}\right]^{\frac{1}{q}}\right. \\
& \left.+\|w\|_{\left[\frac{c_{1}+c_{2}}{2}, c_{2}\right], \infty}\left[\left|\mathbf{u}^{\prime}\left(\boldsymbol{c}_{1}\right)\right|^{q}+3\left|\mathbf{u}^{\prime}\left(\boldsymbol{c}_{2}\right)\right|^{q}\right]^{\frac{1}{q}}\right\} \leq \frac{\left(\boldsymbol{c}_{2}-\boldsymbol{c}_{1}\right)^{v+1}\|w\|_{\left[c_{1}, \boldsymbol{c}_{2}\right], \infty}}{2^{v+1+\frac{2}{q}}(p v+1)^{\frac{1}{p}} \Gamma(v+1)} \\
& \times\left\{\left[3\left|\mathbf{u}^{\prime}\left(c_{1}\right)\right|^{q}+\left|\mathbf{u}^{\prime}\left(c_{2}\right)\right|^{q}\right]^{\frac{1}{q}}+\left[\left|\mathbf{u}^{\prime}\left(c_{1}\right)\right|^{q}+3\left|\mathbf{u}^{\prime}\left(c_{2}\right)\right|^{q}\right]^{\frac{1}{q}}\right\} .
\end{aligned}
$$

(ii) If $\varrho(x)=x$ and $w(x)=1$, then inequality (40) becomes

$$
\begin{aligned}
\mid \frac{2^{v-1} \Gamma(v+1)}{\left(\boldsymbol{c}_{2}-\boldsymbol{c}_{1}\right)^{v}}\left[\left(\frac{\boldsymbol{c}_{1}+\boldsymbol{c}_{2}}{2}\right)+\right. & \left.{ }^{R L} \mathcal{I}^{v} \mathrm{u}\left(\boldsymbol{c}_{2}\right)+{ }^{R L} \mathcal{I}_{\left(\frac{\boldsymbol{c}_{1}+c_{2}}{2}\right)-} \mathrm{u}\left(\boldsymbol{c}_{1}\right)\right]-\mathrm{u}\left(\frac{\boldsymbol{c}_{1}+\boldsymbol{c}_{2}}{2}\right) \mid \\
& \leq \frac{\left(\boldsymbol{c}_{2}-\boldsymbol{c}_{1}\right)^{v+1}\|w\|_{\left[c_{1}, c_{2}\right], \infty}}{2^{v+1+\frac{2}{q}}(p v+1)^{\frac{1}{p}} \Gamma(v+1)} \\
& \times\left\{\left[3\left|\mathrm{u}^{\prime}\left(\boldsymbol{c}_{1}\right)\right|^{q}+\left|\mathrm{u}^{\prime}\left(\boldsymbol{c}_{2}\right)\right|^{q}\right]^{\frac{1}{q}}+\left[\left|\mathrm{u}^{\prime}\left(\boldsymbol{c}_{1}\right)\right|^{q}+3\left|\mathrm{u}^{\prime}\left(\boldsymbol{c}_{2}\right)\right|^{q}\right]^{\frac{1}{q}}\right\},
\end{aligned}
$$

which is already obtained in ([39] [Theorem 6]). 
(iii) If $\varrho(x)=x, w(x)=1$ and $v=1$, then inequality (40) becomes

$$
\begin{aligned}
\mid \frac{1}{c_{2}-c_{1}} \int_{c_{1}}^{c_{2}} \mathrm{u}(x) d x- & \mathrm{u}\left(\frac{\boldsymbol{c}_{1}+\boldsymbol{c}_{2}}{2}\right) \mid \leq \frac{\boldsymbol{c}_{2}-\boldsymbol{c}_{1}}{16}\left(\frac{4}{p+1}\right)^{\frac{1}{p}} \\
& \times\left\{\left[3\left|\mathrm{u}^{\prime}\left(\boldsymbol{c}_{1}\right)\right|^{q}+\left|\mathrm{u}^{\prime}\left(\boldsymbol{c}_{2}\right)\right|^{q}\right]^{\frac{1}{q}}+\left[\left|\mathrm{u}^{\prime}\left(\boldsymbol{c}_{1}\right)\right|^{q}+3\left|\mathrm{u}^{\prime}\left(\boldsymbol{c}_{2}\right)\right|^{q}\right]^{\frac{1}{q}}\right\},
\end{aligned}
$$

which is already obtained in ([45] [Theorem 2.3]).

\section{Concluding Remarks}

In the present article, we have investigated a midpoint fractional HHF integral inequality by using the weighted fractional integrals with positive weighted symmetric function kernels, which is also the midpoint version of (9). Moreover, we have investigated some related results.

The existing versions of HHF integral inequalities (7) and (8) have been successfully applied to other classes of convex functions, see [46-48]. Therefore, our present results can be applied to those classes of convex functions as well.

Furthermore, one can observe that our results in this article are very generic and can be extended to give further potentially useful and interesting HHF integral inequalities of end-midpoint version, like the following one

$$
\begin{aligned}
\mathrm{u}\left(\frac{\mathbf{c}_{1}+\mathbf{c}_{2}}{2}\right) \leq \frac{2^{v-1} \Gamma(v+1)}{\left(\mathbf{c}_{2}-\mathbf{c}_{1}\right)^{v}}\left[{ }^{R L} \mathcal{I}_{\mathbf{c}_{1}+}^{v} \mathrm{u}\left(\frac{\mathbf{c}_{1}+\mathbf{c}_{2}}{2}\right)+{ }^{R L} \mathcal{I}_{\mathbf{c}_{2}-}^{v} \mathrm{u}\left(\frac{\mathbf{c}_{1}+\mathbf{c}_{2}}{2}\right)\right] & \\
& \leq \frac{\mathrm{u}\left(\mathbf{c}_{1}\right)+\mathrm{u}\left(\mathbf{c}_{2}\right)}{2},
\end{aligned}
$$

which was already established by Mohammed and Brevik in [49].

Author Contributions: Conceptualization, P.O.M., H.A., Y.S.H.; methodology, P.O.M., A.K., H.A.; software, P.O.M., A.K., Y.S.H.; validation, P.O.M., A.K., K.M.A., H.A.; formal analysis, P.O.M., A.K., K.M.A.; investigation, P.O.M.; resources, P.O.M., H.A., Y.S.H.; data curation, P.O.M., A.K.; writingoriginal draft preparation, A.K.; writing-review and editing, A.K., P.O.M., H.A.; visualization, A.K., H.A., K.M.A.; supervision, P.O.M., A.K., H.A., Y.S.H. All authors have read and agreed to the final version of the manuscript.

Funding: This research received no external funding.

Institutional Review Board Statement: Not applicable.

Informed Consent Statement: Not applicable.

Data Availability Statement: Not applicable.

Acknowledgments: This work was supported by the Taif University Researchers Supporting Project (No. TURSP-2020/217), Taif University, Taif, Saudi Arabia.

Conflicts of Interest: The authors declare no conflict of interest.

\section{Abbreviations}

The following abbreviations are used in our manuscript:

\footnotetext{
HH Hermite-Hadamard

HHF Hermite-Hadamard-Fejér

RL Riemann-Liouville
} 


\section{References}

1. Hadamard, J. Essay on the study of functions given by their Taylor expansion: Study on the properties of integer functions and in particular of a function considered by Riemann. J. Math. Pures Appl. 1893, 58, 171-215.

2. Zhang, T.-Y.; Ji, A.-P.; Qi, F. Some inequalities of Hermite-Hadamard type for GA-convex functions with applications to means. LeMat 2013, 68, 229-239.

3. Mohammed, P.O.; Vivas-Cortez, M.; Abdeljawad, T.; Rangel-Oliveros, Y. Integral inequalities of Hermite-Hadamard type for quasi-convex functions with applications. AIMS Math. 2020, 5, 7316-7331. [CrossRef]

4. Latif, M.A.; Rashid, S.; Dragomir, S.S.; Chu, Y.M. Hermite-Hadamard type inequalities for co-ordinated convex and quasi-convex functions and their applications. J. Inequal. Appl. 2019, 2019, 317.

[CrossRef]

5. Kashuri, A.; Meftah, B.; Mohammed, P.O. Some weighted Simpson type inequalities for differentiable s-convex functions and their applications. J. Fract. Calc. Nonlinear Syst. 2021, 1, 75-94. [CrossRef]

6. Shi, D.-P.; Xi, B.-Y.; Qi, F. Hermite-Hadamard type inequalities for Riemann-Liouville fractional integrals of $(\alpha, m)-$ convex functions. Fract. Differ. Calc. 2014, 4, 31-43. [CrossRef]

7. Zhou, S.S.; Rashid, S.; Noor, M.A.; Noor, K.I.; Safdar, F.; Chu, Y.M. New Hermite-Hadamard type inequalities for exponentially convex functions and applications. AIMS Math. 2020, 5, 6874-6901. [CrossRef]

8. Rashid, S.; Noor, M.A.; Noor K.I. Fractional exponentially m-convex functions and inequalities. Int. J. Anal. Appl. 2019, 17, 464-478.

9. Mohammed, P.O. Some new Hermite-Hadamard type inequalities for MT-convex functions on differentiable coordinates. J. King Saud Univ. Sci. 2018, 30, 258-262. [CrossRef]

10. Dragomir, S.S.; Pearce, C.E.M. Selected Topics on Hermite-Hadamard Inequalities and Applications; RGMIA Monographs; Victoria University: Footscray, VIC, Australia, 2000.

11. Rashid, S.; Noor, M.A.; Noor, K.I.; Akdemir, A.O. Some new generalizations for exponentially s-convex functions and inequalities via fractional operators. Fractal Fract. 2019, 3, 24. [CrossRef]

12. Kilbas, A.A.; Srivastava, H.M.; Trujillo, J.J. Theory and Applications of Fractional Differential Equations; North-Holland Mathematics Studies; Elsevier Science B.V.: Amsterdam, The Netherlands, 2006; Volume 204.

13. Vanterler, J.; Sousa, C.; Capelas de Oliveira, E. On the $\Psi$-Hilfer fractional derivative. Commun. Nonlinear Sci. Numer. Simul. 2018, 60, 72-91.

[CrossRef]

14. Osler, T.J. The fractional derivative of a composite function. SIAM J. Math. Anal. 1970, 1, 288-293. [CrossRef]

15. Jarad, F.; Abdeljawad, T.; Shah, K. On the weighted fractional operators of a function with respect to another function. Fractals 2020, 28, 12. [CrossRef]

16. Fernandez, A.; Abdeljawad, T.; Baleanu, D. Relations between fractional models with three-parameter Mittag-Leffler kernels. Adv. Differ. Equ. 2020, 2020, 186. [CrossRef]

17. Gavrea, B.; Gavrea, I. On some Ostrowski type inequalities. Gen. Math. 2010, 18, 33-44.

18. Vivas-Cortez, M.; Abdeljawad, T.; Mohammed, P.O.; Rangel-Oliveros, Y. Simpson's integral inequalities for twice differentiable convex functions. Math. Probl. Eng. 2020, 2020, 1936461. [CrossRef]

19. Kaijser, S.; Nikolova, L.; Persson, L.-E.; Wedestig, A. Hardy type inequalities via convexity. Math. Inequal. Appl. 2005, 8, 403-417. [CrossRef]

20. Gunawan, H. Fractional integrals and generalized Olsen inequalities. Kyungpook Math. J. 2009, 49, 31-39. [CrossRef]

21. Sawano, Y.; Wadade, H. On the Gagliardo-Nirenberg type inequality in the critical Sobolev-Morrey space. J. Fourier Anal. Appl. 2013, 19, 20-47. [CrossRef]

22. Mohammed, P.O.; Abdeljawad, T. Opial integral inequalities for generalized fractional operators with nonsingular kernel. J. Inequal. Appl. 2020, 2020, 148. [CrossRef]

23. Sarikaya, M.Z.; Bilisik, C.C.; Mohammed, P.O. Some generalizations of Opial type inequalities. Appl. Math. Inf. Sci. 2020, 14, 809-816.

24. Zhao, C.-J.; Cheung, W.-S. On improvements of the Rozanova's inequality. J. Inequal. Appl. 2011, 2011, 33. [CrossRef]

25. Sarikaya, M.Z.; Set, E.; Yaldiz, H.; Başak, N. Hermite-Hadamard's inequalities for fractional integrals and related fractional inequalities. Math. Comput. Model. 2013, 57, 2403-2407. [CrossRef]

26. Baleanu, D.; Mohammed, P.O.; Zeng, S. Inequalities of trapezoidal type involving generalized fractional integrals. Alex. Eng. J. 2020. [CrossRef]

27. Mohammed, P.O.; Sarikaya, M.Z. Hermite-Hadamard type inequalities for F-convex function involving fractional integrals. J. Inequal. Appl. 2018, 2018, 359. [CrossRef]

28. Qi, F.; Mohammed P.O.; Yao, J.C.; Yao, Y.H. Generalized fractional integral inequalities of Hermite-Hadamard type for $(\alpha, m)-$ convex functions. J. Inequal. Appl. 2019, 2019, 135. [CrossRef]

29. Han, J.; Mohammed, P.O.; Zeng, H. Generalized fractional integral inequalities of Hermite-Hadamard-type for a convex function. Open Math. 2020, 18, 794-806. [CrossRef]

30. Mohammed, P.O.; Abdeljawad, T.; Zeng, S.; Kashuri, A. Fractional Hermite-Hadamard integral inequalities for a new class of convex functions. Symmetry 2020, 12, 1485. [CrossRef] 
31. Mohammed, P.O.; Abdeljawad, T.; Alqudah, M.A.; Jarad, F. New discrete inequalities of Hermite-Hadamard type for convex functions. Adv. Differ. Equ. 2021, 2021, 122. [CrossRef]

32. Baleanu, D.; Mohammed, P.O.; Vivas-Cortez, M.; Rangel-Oliveros, Y. Some modifications in conformable fractional integral inequalities. Adv. Differ. Equ. 2020, 2020, 374.

[CrossRef]

33. Abdeljawad, T.; Mohammed, P.O.; Kashuri, A. New modified conformable fractional integral inequalities of Hermite-Hadamard type with applications. J. Funct. Spaces 2020, 2020, 4352357. [CrossRef]

34. Baleanu, D.; Kashuri, A.; Mohammed, P.O.; Meftah, B. General Raina fractional integral inequalities on coordinates of convex functions. Adv. Differ. Equ. 2021, 2021, 82. [CrossRef]

35. Mohammed, P.O.; Abdeljawad, T. Integral inequalities for a fractional operator of a function with respect to another function with nonsingular kernel. Adv. Differ. Equ. 2020, 2020, 363. [CrossRef]

36. Mohammed, P.O. Hermite-Hadamard inequalities for Riemann-Liouville fractional integrals of a convex function with respect to a monotone function. Math. Meth. Appl. Sci. 2019, 1-11.

[CrossRef]

37. Mohammed, P.O.; Sarikaya, M.Z.; Baleanu, D. On the generalized Hermite-Hadamard inequalities via the tempered fractional integrals. Symmetry 2020, 12, 595. [CrossRef]

38. Fernandez, A.; Mohammed. P. Hermite-Hadamard inequalities in fractional calculus defined using Mittag-Leffler kernels. Math Meth. Appl. Sci. 2020,1-18. [CrossRef]

39. Sarikaya, M.Z.; Yildirim, H. On Hermite-Hadamard type inequalities for Riemann-Liouville fractional integrals. Miskolc Math. Notes 2017, 17, 1049-1059. [CrossRef]

40. Macdonald, I.G. Symmetric Functions and Orthogonal Polynomials; American Mathematical Society: New York, NY, USA, 1997.

41. Fejér, L. Uberdie Fourierreihen, II, Math. Naturwise Anz Ungar. Akad. Wiss. 1906, 24, 369-390.

42. Işcan, İ. Hermite-Hadamard-Fejér type inequalities for convex functions via fractional integrals. Stud. Univ. Babeş Bolyai Math. 2015, 60, 355-366.

43. Set, E; Işcan, İ.; Sarikaya, M.Z.; Özdemir, M. E. On new inequalities of Hermite-Hadamard-Fejér type for convex functions via fractional integrals. Appl. Math. Comput. 2015, 259, 875-881.

[CrossRef]

44. Mohammed, P.O.; Abdeljawad, T.; Kashuri, A. Fractional Hermite-Hadamard-Fejér inequalities for a convex function with respect to an increasing function involving a positive weighted symmetric function. Symmetry 2020, 12, 1503. [CrossRef]

45. Kırmac1, U. Inequalities for differentiable mappings and applications to special means of real numbers to midpoint formula. Appl. Math. Comput. 2004, 147, 137-146.

46. Kunt, M.; Işcan, İ. On new Hermite-Hadamard-Fejér type inequalities for $p$-convex functions via fractional integrals. Commun. Math. Model. Appl. 2017, 2, 1-15. [CrossRef]

47. Delavar, M.R.; Aslani, M.; De La Sen, M. Hermite-Hadamard-Fejér inequality related to generalized convex functions via fractional integrals. Sci. Asia 2018, 2018, 5864091.

48. Mehmood, S.; Zafar, F.; Asmin, N. New Hermite-Hadamard-Fejér type inequalities for $\left(\eta_{1}, \eta_{2}\right)$-convex functions via fractional calculus. Sci. Asia 2020, 46, 102-108.

[CrossRef]

49. Mohammed, P.O.; Brevik, I. A new version of the Hermite-Hadamard inequality for Riemann-Liouville fractional integrals. Symmetry 2020, 12, 610. [CrossRef] 